TRANSACTIONS OF THE

AMERICAN MATHEMATICAL SOCIETY

Volume 363, Number 7, July 2011, Pages 3853-3892

S 0002-9947(2011)05352-3

Article electronically published on February 10, 2011

\title{
APPROXIMATION OF QUASI-POTENTIALS AND EXIT PROBLEMS FOR MULTIDIMENSIONAL RDE'S WITH NOISE
}

\author{
SANDRA CERRAI AND MARK FREIDLIN
}

\begin{abstract}
We deal with a class of reaction-diffusion equations, in space dimension $d>1$, perturbed by a Gaussian noise $\partial w^{\delta} / \partial t$ which is white in time and colored in space. We assume that the noise has a small correlation radius $\delta$, so that it converges to the white noise $\partial w / \partial t$, as $\delta \downarrow 0$. By using arguments of $\Gamma$-convergence, we prove that, under suitable assumptions, the quasi-potential $V_{\delta}$ converges to the quasi-potential $V$, corresponding to spacetime white noise, in spite of the fact that the equation perturbed by space-time white noise has no solution.

We apply these results to the asymptotic estimate of the mean of the exit time of the solution of the stochastic problem from a basin of attraction of an asymptotically stable point for the unperturbed problem.
\end{abstract}

\section{INTRODUCTION}

Consider a dynamical system in $\mathbb{R}^{d}$, perturbed by noise

$$
\dot{X}_{\epsilon}^{x}(t)=f\left(X_{\epsilon}^{x}(t)\right)+\sqrt{\epsilon} \dot{w}(t), \quad X_{\epsilon}^{x}(0)=x \in \mathbb{R}^{d} .
$$

Here $f(x)$ is a Lipschitz continuous vector field, $w(t)$ is the Wiener process on $\mathbb{R}^{d}$ and $\epsilon>0$ is a small parameter. Now, let $O \in \mathbb{R}^{d}$ be an asymptotically stable equilibrium for $X_{0}^{x}(t)$ and let $D$ be a bounded domain in $\mathbb{R}^{d}$ such that $D \cup \partial D$ is attracted to $O$.

It is clear that, under these assumptions, for any $x \in D$ the exit of $X_{\epsilon}^{x}(t)$ from $D$ occurs due to the large deviations of $X_{\epsilon}^{x}(t)$ from $X_{0}^{x}(t)$. As is known, the large deviation action functional for the family $\left\{X_{\epsilon}^{x}\right\}_{\epsilon>0}$, in the space $C\left([0, T] ; \mathbb{R}^{d}\right)$, is given by $\epsilon^{-1} S_{0, T}$, where

$$
S_{0, T}(\varphi)= \begin{cases}\frac{1}{2} \int_{0}^{T}|\dot{\varphi}(s)-f(\varphi(s))|^{2} d s, & \text { if } \varphi \text { is absolutely continuous } \\ +\infty, & \text { otherwise }\end{cases}
$$

(see [10] for all details).

Moreover, the quasi-potential for the field $f$, with respect to the equilibrium $O$ and the perturbation $\sqrt{\epsilon} \dot{w}$, is defined as

$$
V(x):=\inf \left\{S_{0, T}(\varphi): \varphi(0)=O, \varphi(T)=x, T \geq 0\right\} .
$$

If the field $f$ is potential; that is $f(x)=-\nabla U(x)$, with $U(O)=0, U(x)>0$ and $\nabla U(x) \neq 0$, for any $x \neq O$, then the quasi-potential coincides, up to a constant

Received by the editors June 5, 2009 and, in revised form, March 19, 2010.

2010 Mathematics Subject Classification. Primary 60H15, 60F10, 35K57, 49J45.

The second author was partially supported by an NSF grant.

(C)2011 American Mathematical Society 
factor, with the potential; that is

$$
V(x)=2 U(x), \quad x \in\left\{y \in D \cap \partial D: U(y) \leq \inf _{z \in \partial D} U(z)\right\} .
$$

Moreover, if we define

$$
\tau_{\epsilon}^{x}:=\inf \left\{t \geq 0: X_{\epsilon}^{x}(t) \notin D\right\},
$$

then, as it is shown in [10],

$$
\lim _{\epsilon \rightarrow 0} \epsilon \log \mathbb{E} \tau_{\epsilon}^{x}=\inf _{z \in \partial D} V(z),
$$

and, when the infimum above is achieved at only one point $x_{0} \in \partial D$, it holds that

$$
\lim _{\epsilon \rightarrow 0} X_{\epsilon}^{x}\left(\tau_{\epsilon}^{x}\right)=x_{0} .
$$

In view of these facts, the quasi-potential allows us to answer many questions related to the exit problem. The quasi-potential also allows us to describe transactions between stable attractors if the system has many of them. Furthermore, the fact that

$$
\inf \left\{S_{0, T}(\varphi): \varphi(0)=x, \varphi(T)=y\right\} \leq c|x-y|, \quad T=|x-y|,
$$

implies that $V(x)$ is Lipschitz continuous, and this allows us, in particular, to carry out important bounds.

Now consider perturbations of a reaction-diffusion equation by the space-time white noise

$$
\begin{aligned}
& \frac{\partial u_{\epsilon}}{\partial t}(t, \xi)=\frac{\partial^{2} u_{\epsilon}}{\partial \xi^{2}}(t, \xi)-\lambda u_{\epsilon}(t, \xi)+b\left(\xi, u_{\epsilon}(t, \xi)\right)+\sqrt{\epsilon} \frac{\partial w}{\partial t}(t, \xi), \quad t>0, \quad \xi \in[0, L], \\
& u_{\epsilon}(t, 0)=u_{\epsilon}(t, 0)=0, \quad u_{\epsilon}(0, \xi)=x(\xi) .
\end{aligned}
$$

Here $\partial w / \partial t$ is a space-time white noise, $\lambda>0$ and the function $b$ is assumed to be Lipschitz continuous in $\xi$, with $f(\xi, 0)=0$ and $[b(\xi, \cdot)]_{\operatorname{Lip}}<\lambda$ for any $\xi \in[0, L]$.

It is clear that $\bar{x}(\xi) \equiv 0$ is an asymptotical stable equilibrium of the nonperturbed semiflow $u_{0}(t, \xi)$. If $D$ is a domain in the functional space, attracted with its boundary to $\bar{x}(\xi) \equiv 0$, one can consider the exit problem from $D$ for $u_{\epsilon}^{x}(t, \xi)$, with $0<\epsilon \ll 1$. In this case, the normalized action functional $S_{0, T}(u)$ for the family $u_{\epsilon}^{x}(t, \xi)$, as $\epsilon \downarrow 0$, has the form

$$
S_{0, T}(u)=\frac{1}{2} \int_{0}^{T} \int_{0}^{L}\left[\frac{\partial u}{\partial t}(s, \xi)-\frac{\partial^{2} u}{\partial \xi^{2}}(s, \xi)+\lambda u(s, \xi)-b(\xi, u(s, \xi))\right]^{2} d \xi d s
$$

for $u$ in the appropriate Sobolev space and

$$
S_{0, T}(u)=+\infty
$$

for $u$ in the rest of the space of square integrable functions.

The quasi-potential in this case is given by the formula

$$
V(x)=\frac{1}{2} \int_{0}^{1}\left[\left(\frac{d x}{d \xi}\right)^{2}+\lambda x^{2}-\mathcal{B}(\xi, x)\right] d \xi, \quad x \in W^{1,2}(0, L),
$$

where

$$
\mathcal{B}(\xi, \sigma)=2 \int_{0}^{\sigma} b(\xi, r) d r .
$$


Moreover,

$$
\frac{\delta V}{\delta x}(x)=-\frac{d^{2} x}{d \xi^{2}}+\lambda x-b(\cdot, x),
$$

so that the non-perturbed flow $u_{0}(t, \xi)$, defined by equation (1.2) with $\epsilon=0$, is of gradient type. This in particular allows us to study the behaviour of the invariant measure for $u^{\epsilon}$, as $\epsilon \downarrow 0$.

As is known, in the case of a multidimensional space variable $\xi$, the equation (1.3)

$$
\left\{\begin{array}{l}
\frac{\partial u_{\epsilon}}{\partial t}(t, \xi)=(\Delta-\lambda) u_{\epsilon}(t, \xi)+b\left(\xi, u_{\epsilon}(t, \xi)\right)+\sqrt{\epsilon} \frac{\partial w}{\partial t}(t, \xi), \quad t>0, \quad x \in \mathcal{O} \subset \mathbb{R}^{d} \\
\mathcal{N} u_{\epsilon}(t, \xi)=0, \quad \xi \in \partial \mathcal{O}, \quad u_{\epsilon}(0, \xi)=x(\xi)
\end{array}\right.
$$

with appropriate boundary or periodicity conditions, is not solvable in general for space-time white noise $\partial w / \partial t$. To consider equation (1.3), with $d>1$, one can assume that $\partial w / \partial t$ is white in time and has some smoothness in the space variable $\xi$. Then, one can prove existence and uniqueness for equation (1.3), with corresponding boundary conditions, and then calculate the action functional.

The action functional in this case is non-local in $\xi$ and is not as explicit as in white noise case. The quasi-potential, defined as the solution of the corresponding variational problem, is also more complicated. Moreover, the quasi-potential is finite only on a smaller Sobolev space. This leads to some difficulties in the identification of the limiting exit point and in the calculation of the asymptotics of the exit times through the quasi-potential. These difficulties are, mostly, due to the difficulties with the controllability of the non-perturbed flow. But even if we overcome these problems, the complicated form of the quasi-potential leads to non-standard variational problems.

On the other hand, one can assume that the noise $\partial w / \partial t$ has a small correlation radius $\delta$, that is $\partial w / \partial t=\partial w^{\delta} / \partial t$, so that $\partial w^{\delta} / \partial t$ converges to the space-time white noise, as $\delta \downarrow 0$. Thus, one can expect that, under suitable assumptions, the quasi-potential $V_{\delta}(x)$ for the equation with regularized noise converges to the quasi-potential $V(x)$, corresponding to the space-time white noise in (1.3), in spite of the fact that (1.3) cannot be solved for space-time white noise. In such a way, the classical functional of calculus of variation $V(x)$ characterizes the asymptotics of the exit time $\tau_{\epsilon, \delta}^{x}$ from a bounded domain $D$ of the space $L^{2}(\mathcal{O})$, for $0<\epsilon \ll \delta \ll 1$.

In the present paper we are dealing with the following family of stochastic reaction-diffusion equations:

$$
\left\{\begin{array}{l}
\frac{\partial u}{\partial t}(t, \xi)=\mathcal{A} u(t, \xi)+b(\xi, u(t, \xi))+\sqrt{\epsilon} g(\xi, u(t, \xi)) \frac{\partial w^{Q_{\delta}}}{\partial t}(t, \xi), \\
u(0, \xi)=x(\xi), \quad \mathcal{N} u(t, \xi)=0, \quad \xi \in \partial \mathcal{O}
\end{array}\right.
$$

in a bounded domain $\mathcal{O} \subset \mathbb{R}^{d}$, with $d \geq 1$, having a regular boundary, depending on a small parameter $\epsilon>0$. Here, $\mathcal{A}$ is the second order differential operator

$$
\mathcal{A}(\xi)=\sum_{i, j=1}^{d} \frac{\partial}{\partial \xi_{i}}\left(a_{i j}(\xi) \frac{\partial}{\partial \xi_{j}}\right)-\lambda, \quad \xi \in \mathcal{O} .
$$


The constant $\lambda$ is strictly positive, the coefficients $a_{i j}$ are of class $C^{1}(\mathcal{O})$ and a uniform ellipticity condition holds for the matrix $a(\xi):=\left[a_{i j}(\xi)\right]_{i, j=1}^{d}$; that is,

$$
\inf _{\xi \in \overline{\mathcal{O}}}\langle a(\xi) h, h\rangle \geq \alpha_{0}|h|^{2}, \quad h \in \mathbb{R}^{d},
$$

for some strictly positive constant $\alpha_{0}$. Finally, the operator $\mathcal{N}$ acts on $\partial \mathcal{O}$ and is assumed to be either of Dirichlet or of co-normal type; that is,

$$
\mathcal{N} f(\xi)=f(\xi), \quad \text { or } \quad \mathcal{N} f(\xi)=\langle a(\xi) \nu(\xi), \nabla f(\xi)\rangle, \quad \xi \in \partial \mathcal{O},
$$

where $\nu(\xi)$ is the unit normal vector at the point $\xi \in \partial \mathcal{O}$.

The stochastic perturbation is given by a Gaussian noise $\partial w^{Q_{\delta}} / \partial t(t, \xi)$, for $t \geq 0$ and $\xi \in \mathcal{O}$, which is assumed to be white in time and colored in space, in the case of space dimension $d>1$. Formally, the cylindrical Wiener process $w^{Q_{\delta}}(t, \xi)$ is defined as the infinite sum

$$
w^{Q_{\delta}}(t, \xi)=\sum_{k=1}^{\infty} Q_{\delta} e_{k}(\xi) \beta_{k}(t),
$$

where $\left\{e_{k}\right\}_{k \in \mathbb{N}}$ is a complete orthonormal basis in $L^{2}(\mathcal{O}),\left\{\beta_{k}(t)\right\}_{k \in \mathbb{N}}$ is a sequence of mutually independent standard Brownian motions defined on the same complete stochastic basis $\left(\Omega, \mathcal{F}, \mathcal{F}_{t}, \mathbb{P}\right)$ and $Q_{\delta}$ is a linear operator on $L^{2}(\mathcal{O})$. In what follows we shall assume that in the case of space dimension $d \geq 2$

$$
Q_{\delta} \sim(-A)^{-\beta}, \quad \text { with } \beta>\frac{d-2}{4},
$$

where $A$ is the realization of the operator $\mathcal{A}$ in $L^{2}(\mathcal{O})$, endowed with the boundary conditions $\mathcal{N}$. Moreover, we shall assume that the operator $Q_{\delta}$ converges strongly to the identity operator, as $\delta \downarrow 0$ (for all details see Hypothesis 1 and Remark 2.1).

Finally, for the coefficients $f$ and $g$ we assume smoothness and boundedness of derivatives and some compatibility conditions with the boundary conditions (1.6). Moreover, we assume that the diffusion coefficient $g$ is non-degenerate and $b(\xi, 0)=$ 0 , for all $\xi \in \mathcal{O}$ (for all details see Hypothesis 2 and Remark 2.2).

Under our assumptions, for any $\delta, \epsilon>0$ and $x \in L^{2}(\mathcal{O})$ problem (1.4) admits a unique mild solution $u_{\epsilon, \delta}^{x} \in L^{p}\left(\Omega ; C\left((0, T] ; L^{2}(\mathcal{O})\right)\right)$, with $T>0$ and $p \geq 1$. For any $\delta>0$, the good large deviation action functional for the family $\left\{\mathcal{L}\left(u_{\epsilon, \delta}^{x}\right)\right\}_{\epsilon>0}$ in $C\left((0, T] ; L^{2}(\mathcal{O})\right)$ is given by $\epsilon^{-1} S_{0, T}^{\delta}$, where

$$
S_{T_{1}, T_{2}}^{\delta}(u)=\frac{1}{2} \int_{T_{1}}^{T_{2}}\left|Q_{\delta}^{-1}\left(\frac{\partial_{t} u(t)-\mathcal{A} u(t)-b(\cdot, u(t))}{g(\cdot, u(t))}\right)\right|_{L^{2}(\mathcal{O})}^{2} d t,
$$

for $-\infty \leq T_{1}<T_{2} \leq+\infty$ and $u$ in the appropriate Sobolev space, and $S_{T_{1}, T_{2}}^{\delta}(u)=$ $+\infty$, for $u$ in what remains of $C\left(\left[T_{1}, T_{2}\right] ; L^{2}(\mathcal{O})\right)$. The corresponding quasi-potential is defined by

$$
V_{\delta}(x)=\inf \left\{S_{0, T}^{\delta}(u): u \in C\left([0, T] ; L^{2}(\mathcal{O})\right), u(0)=0, u(T)=x, T>0\right\},
$$

for any $x \in L^{2}(\mathcal{O})$.

When $d=1$ and $g \equiv 1$, (1.4) is a gradient system and the quasi-potential can be explicitly computed as

$$
V(x)=\frac{1}{2} \int_{\mathcal{O}}\left|(-A)^{\frac{1}{2}} x(\xi)\right|^{2} d \xi-\int_{\mathcal{O}} \int_{0}^{x(\xi)} b(\xi, \sigma) d \sigma d \xi .
$$


The expression in (1.8) coincides with the quasi-potential in dimension $d=1$ only, but it is perfectly meaningful in any space dimension. Moreover, it is possible to prove that

$$
V(x)=\inf \left\{S_{0, T}(u): u \in C\left([0, T] ; L^{2}(\mathcal{O})\right), u(0)=0, u(T)=x, T>0\right\},
$$

where $S_{0, T}(u)$ is the fuctional obtained from $S_{0, T}^{\delta}$, with $Q_{\delta}$ replaced by the identity operator (for a proof see e.g. [6, Theorem 12.23]). In this paper our aim is to prove that $V$ approximates $V_{\delta}$, as the small parameter $\delta>0$ approaches zero, in any space dimension $d$. More precisely, we want to show that

$$
\lim _{\delta \rightarrow 0} V_{\delta}(x)=V(x), \quad x \in D\left((-A)^{\beta+\frac{1}{2}}\right) .
$$

In [5], $V_{\delta}$ has been characterized as

$$
V_{\delta}(x)=\min \left\{S_{-\infty, 0}^{\delta}(u): u \in \mathcal{X}_{x}\right\}
$$

where

$$
\mathcal{X}_{x}:=\left\{u \in C\left((-\infty, 0] ; L^{2}(\mathcal{O})\right), \lim _{t \rightarrow-\infty}|u(t)|_{L^{2}(\mathcal{O})}=0, u(0)=x\right\} .
$$

Then, $V$ can also be characterized as

$$
V(x)=\min \left\{S_{-\infty, 0}(u): u \in \mathcal{X}_{x}\right\},
$$

where $S_{-\infty, 0}$ is the same as $S_{-\infty, 0}^{\delta}$, when $Q_{\delta}=I$. It is natural to study limit (1.9) by using a $\Gamma$-convergence argument (for all definitions and results on $\Gamma$-convergence see Section [3, where we have collected some results from [8]). To this purpose, in Section 5 we show that

$$
\Gamma-\lim _{\delta \rightarrow 0} S_{-\infty, 0}^{\delta}=S_{-\infty, 0}, \quad \text { in } \mathcal{X}_{x},
$$

for any $x \in D\left((-A)^{\beta+\frac{1}{2}}\right)$ and, since the family $\left\{S_{-\infty, 0}^{\delta}\right\}_{\delta>0}$ is equi-coercive, this implies that the minima of $S_{-\infty, 0}^{\delta}$ on $\mathcal{X}_{x}$ converge to the minimum of $S_{-\infty, 0}$ on $\mathcal{X}_{x}$, as $\delta \downarrow 0$. The proof of the $\Gamma$-convergence of $S_{-\infty, 0}^{\delta}$ to $S_{-\infty, 0}$ is not trivial and requires a detailed analysis of the skeleton equation associated with problem (1.4), based on optimal regularity and controllability of degenerate systems.

As an example of the possible applications of limit (1.9), in Section[7we introduce the problem of the exit from a basin of attraction $D$ for the solution of (1.4) and we prove that, as in the finite dimensional case, the mean of the exit time $\tau_{\epsilon, \delta}^{x}$ from $D$ is asymptotically equivalent to

$$
\exp \left(\frac{1}{\epsilon} \inf _{y \in \partial D} V_{\delta}(y)\right)
$$

Moreover, in view of (1.9), by again using $\Gamma$-convergence, we prove that under suitable conditions on $\partial D$

$$
\lim _{\delta \rightarrow 0} \inf _{x \in \partial D} V_{\delta}(x)=\inf _{x \in \partial D} V(x) .
$$

In particular, this implies that

$$
\mathbb{E} \tau_{\epsilon, \delta}^{x} \approx \exp \left(\frac{1}{\epsilon} \inf _{x \in \partial D} V(x)\right)
$$

as $0<\epsilon \ll \delta \ll 1$, and what is relevant is that in many situations the expression on the right hand side can be explicitly computed, depending on the domain $D$. We would like to stress that the proof of the asymptotic characterization of the 
mean of the exit time from the domain $D$ in terms of $\inf _{y \in \partial D} V_{\delta}(y)$ is delicate, also because in this infinite dimensional and degenerate framework the quasi-potential is not Lipschitz continuous and the proof of the key lemma, Lemma 7.2, requires some substantial new work.

\section{Set-uP}

Let $\mathcal{O}$ be a bounded open subset of $\mathbb{R}^{d}$, with $d \geq 1$, having a regular boundary. We denote by $H$ the Hilbert space $L^{2}(\mathcal{O})$, endowed with the usual scalar product $\langle\cdot, \cdot\rangle_{H}$ and the corresponding norm $|\cdot|_{H}$. For any $p \geq 1$, we denote by $|\cdot|_{p}$ the usual norm in $L^{p}(\mathcal{O})$.

For any $1 \leq p \leq \infty$ and $m \in \mathbb{N}$, we denote by $W^{m, p}(\mathcal{O})$ the space of functions $f \in L^{p}(\mathcal{O})$ such that the weak derivatives $D^{\alpha} f$ exist in $L^{p}(\mathcal{O})$, for each $0 \leq|\alpha| \leq m$. $W^{m, p}(\mathcal{O})$ is a Banach space, endowed with the norm

$$
|f|_{W^{m, p}(\mathcal{O})}:=\sum_{|\alpha| \leq m}\left|D^{\alpha} f\right|_{L^{p}(\mathcal{O})} .
$$

Moreover, if $s>0$ is not an integer, we define $W^{s, p}(\mathcal{O})$ as the space of functions $f \in W^{[s], p}(\mathcal{O})$ such that

$$
|f|_{W^{s, p}(\mathcal{O})}:=|f|_{W^{[s], p}(\mathcal{O})}+\sum_{|\alpha|=[s]} \int_{\varnothing \times \varnothing} \frac{\left|D^{\alpha} f(\xi)-D^{\alpha} f(\eta)\right|^{p}}{|\xi-\eta|^{d+(s-[s]) p}} d \xi d \eta<\infty .
$$

Next, we recall that for any $s \in \mathbb{R}$ and $p \in(1, \infty)$ the Bessel potential space $H^{s, p}\left(\mathbb{R}^{d}\right)$ is defined by

$$
H^{s, p}\left(\mathbb{R}^{d}\right):=\left\{f \in \mathcal{S}^{\prime}\left(\mathbb{R}^{d}\right):|f|_{H^{s, p}\left(\mathbb{R}^{d}\right)}:=\left|\mathcal{F}^{-1}\left(1+|\xi|^{2}\right)^{s / 2} \mathcal{F} f\right|_{L^{p}\left(\mathbb{R}^{d}\right)}<\infty\right\},
$$

where $\mathcal{S}^{\prime}\left(\mathbb{R}^{d}\right)$ is the space of tempered distributions on $\mathbb{R}^{d}$ and $\mathcal{F}$ is the Fourier transform. The Bessel potential spaces on $\mathcal{O}$ are defined by restriction, as

$$
H^{s, p}(\mathcal{O}):=\left\{f=g_{\left.\right|_{\partial \mathcal{O}}} ; g \in H^{s, p}\left(\mathbb{R}^{d}\right)\right\},
$$

with

$$
|f|_{H^{s, p}(\mathcal{O})}:=\inf _{f=g_{\left.\right|_{\partial \mathcal{O}}}}|g|_{H^{s, p}\left(\mathbb{R}^{d}\right)} .
$$

Note that for $k \in \mathbb{N}$ the Bessel space $H^{k, p}(\mathcal{O})$ coincides with the Sobolev space $W^{k, p}(\mathcal{O})$ (for all definitions and detailed proofs we refer to [14]).

Concerning the multiplications of functions in Bessel spaces, we recall that as proved e.g. in [12, Theorem 4.6.1.1], if $s_{1} \leq s_{2}$ and $s_{1}+s_{2}>0$,

$$
s_{2}>\frac{d}{2} \Longrightarrow H^{s_{1}, 2}(\mathcal{O}) \cdot H^{s_{2}, 2}(\mathcal{O}) \hookrightarrow H^{s_{1}, 2}(\mathcal{O}) .
$$

Finally, for any $-\infty \leq T_{1}<T_{2} \leq+\infty$ we shall denote by $W_{2}^{1,2}\left(\left(T_{1}, T_{2}\right) \times \mathcal{O}\right)$ the space of functions

$$
u \in L^{2}\left(\left(T_{1}, T_{2}\right) \times \mathcal{O}\right)
$$

such that $D_{t} u$ and $D_{x}^{\alpha}$ exist in $L^{2}\left(\left(T_{1}, T_{2}\right) \times \mathcal{O}\right)$, for any $1 \leq|\alpha| \leq 2$. $W_{2}^{1,2}\left(\left(T_{1}, T_{2}\right) \times\right.$ $\mathcal{O})$ is a Banach space, endowed with the norm

$|u|_{W_{2}^{1,2}\left(\left(T_{1}, T_{2}\right) \times \mathcal{O}\right)}:=|u|_{L^{2}\left(\left(T_{1}, T_{2}\right) \times \mathcal{O}\right)}+\left|D_{t} u\right|_{L^{2}\left(\left(T_{1}, T_{2}\right) \times \mathcal{O}\right)}+\sum_{|\alpha| \leq 2}\left|D_{x}^{\alpha} u\right|_{L^{2}\left(\left(T_{1}, T_{2}\right) \times \mathcal{O}\right)}$.

In what follows, we shall denote by $A$ the realization in $H$ of the operator $\mathcal{A}$ introduced in (1.5), endowed with the boundary condition $\mathcal{N}$ described in (1.6). It 
is well known that $A$ is a self-adjoint operator generating an analytic semigroup on $H$, which we shall denote by $e^{t A}$. As proved e.g. in [14, Chapter 5], we have

$$
D(A)=\left\{f \in H^{2,2}(\mathcal{O}): \mathcal{N} f=0\right\}=: H_{\mathcal{N}}^{2,2}(\mathcal{O})
$$

and, for any $l \in \mathbb{N}^{+}$, the following optimal regularity result holds:

$$
f \in D(A), \quad A f \in H^{l, 2}(\mathcal{O}) \Rightarrow f \in H^{l+2,2}(\mathcal{O}) .
$$

For any $\gamma \geq 0$, we introduce the following boundary operator:

$$
\mathcal{N}_{\gamma}:= \begin{cases}\emptyset & \text { if } \gamma \in[0, m] \\ \left\{\mathcal{N}, \mathcal{N} \mathcal{A}, \ldots, \mathcal{N} \mathcal{A}^{k}\right\} & \text { if } \gamma \in(k+m, k+1+m], \quad k=0,1, \ldots,\end{cases}
$$

where

$$
m:=\frac{1+2 \operatorname{ord} \mathcal{N}}{4}= \begin{cases}\frac{1}{4}, & \text { if } \mathcal{N}=I, \\ \frac{3}{4}, & \text { if } \mathcal{N}=\langle a \nu, \nabla\rangle .\end{cases}
$$

In [5, Proposition 2.1] we have seen that for any $\gamma>0$

$$
D\left((-A)^{\gamma}\right)=H_{\mathcal{N}_{\gamma}}^{2 \gamma, 2}(\mathcal{O}) .
$$

As is known, the semigroup $e^{t A}$ has a smoothing effect. Namely, for any $t>0$, $1 \leq q \leq p \leq \infty$ and $\epsilon \geq 0, e^{t A}$ maps $L^{q}(\mathcal{O})$ into $W^{\epsilon, p}(\mathcal{O})$ and

$$
\left|e^{t A} x\right|_{W^{\epsilon, p}(\mathcal{O})} \leq c e^{-\lambda t}(t \wedge 1)^{-\left(\frac{\epsilon}{2}+\frac{d(p-q)}{2 p q}\right)}|x|_{q}, \quad x \in L^{q}(\mathcal{O}) .
$$

Moreover, as $A$ is self-adjoint and the domain $\mathcal{O}$ satisfies nice properties, there exists a complete orthonormal system $\left\{e_{k}\right\}_{k \in \mathbb{N}}$ in $H$ and a sequence of positive real numbers $\left\{\alpha_{k}\right\}_{k \in \mathbb{N}}$ such that

$$
A e_{k}=-\alpha_{k} e_{k}, \quad k \in \mathbb{N},
$$

and

$$
\alpha_{k} \approx k^{\frac{2}{d}} .
$$

Finally, due to the fact that $A$ is dissipative, it is immediate to check that for any $h \in L^{2}\left(\left(T_{1}, T_{2}\right) ; D\left((-A)^{\alpha}\right)\right)$, with $\alpha \geq 0$ and $T_{1}<T_{2}$, it holds that

$$
\left|\int_{T_{1}}^{T_{2}} e^{\left(T_{2}-s\right) A} h(s) d s\right|_{D\left((-A)^{\alpha+\frac{1}{2}}\right)} \leq c_{\alpha}|h|_{L^{2}\left(\left(T_{1}, T_{2}\right) ; D\left((-A)^{\alpha}\right)\right)},
$$

for some constant $c_{\alpha}$ independent of $T_{1}$ and $T_{2}$.

In what follows we shall assume that, for any $\delta>0$, the covariance operator $Q_{\delta}$ of the Gaussian noise $w^{Q_{\delta}}$ (see (1.7)) satisfies the following conditions.

\section{Hypothesis 1.}

1. The operator $Q_{\delta}$ is diagonal with respect to the basis $\left\{e_{k}\right\}_{k \in \mathbb{N}}$ which diaginalizes A, with strictly positive eigenvalues $\left\{\lambda_{\delta, k}\right\}_{k \in \mathbb{N}}$. Moreover, if $d>1$, we have

$$
\frac{1}{c_{\delta}} \alpha_{k}^{-\beta} \leq \lambda_{\delta, k} \leq c_{\delta} \alpha_{k}^{-\beta}, \quad k \in \mathbb{N},
$$

for some constant $c_{\delta}>0$, independent of $k \in \mathbb{N}$, and some

$$
\beta>\frac{d-2}{4} .
$$


2. For any $0<\delta_{1}<\delta_{2}$ we have

$$
\lambda_{\delta_{1}, k} \geq \lambda_{\delta_{2}, k}, \quad k \in \mathbb{N} .
$$

Moreover,

$$
\lim _{\delta \rightarrow 0} \lambda_{\delta, k}=1, \quad k \in \mathbb{N} .
$$

Remark 2.1.

1. Since we have $\alpha_{k} \approx k^{2 / d}$ and $\beta>(d-2) / 4$, as a consequence of (2.9) it is possible to prove that for any $d>1$

there exists $\left\{\begin{array}{ll}\gamma<\infty & \text { if } d=2 \\ \gamma<\frac{2 d}{d-2} & \text { if } d>2\end{array}\right.$ such that $\left\|Q_{\delta}\right\|_{\gamma}:=\left(\sum_{k=1}^{\infty} \lambda_{\delta, k}^{\gamma}\right)^{1 / \gamma}<\infty$.

In particular, notice that, as $2 d /(d-2)>2$, we are not assuming $Q_{\delta}$ to be a Hilbert-Schmidt operator.

2. If we set

$$
\lambda_{\delta, k}=\left(1+\delta \alpha_{k}^{\beta}\right)^{-1}
$$

then conditions (2.9), (2.11) and (2.12) are all trivially satisfied.

3. Due to (2.9), we have that

$$
\operatorname{Ker} Q_{\delta}=\{0\}, \quad \delta>0,
$$

and for any $\delta_{1}, \delta_{2}>0$

$$
\operatorname{Im} Q_{\delta_{1}}=\operatorname{Im} Q_{\delta_{2}}=D\left((-A)^{\beta}\right),
$$

with

$$
\left|(-A)^{\beta} Q_{\delta} x\right|_{H} \leq c_{\delta}|x|_{H}, \quad x \in H,
$$

and

$$
\left|Q_{\delta}^{-1} x\right|_{H} \leq c_{\delta}|x|_{D\left((-A)^{\beta}\right)}, \quad x \in D\left((-A)^{\beta}\right) .
$$

This implies that $Q_{\delta}^{-1} y$ is well defined, for any $\delta>0$ and $y \in \operatorname{Im} Q_{\delta}$, and thanks to (2.9) and (2.11), for any $0<\delta_{<} \delta_{2}$

$$
\left|Q_{\delta_{1}}^{-1} y\right|_{H} \leq\left|Q_{\delta_{2}}^{-1} y\right|_{H} \leq c_{\delta_{1}}^{-1} c_{\delta_{2}}\left|Q_{\delta_{1}}^{-1} y\right|_{H}
$$

4. According to (2.12) we have that

$$
\lim _{\delta \rightarrow 0} Q_{\delta} x=x, \quad x \in H, \quad \lim _{\delta \rightarrow 0} Q_{\delta}^{-1} x=x, \quad x \in \operatorname{Im} Q_{1} .
$$

Moreover, we have

$$
\left|Q_{\delta}^{-1} x\right|_{H} \geq|x|_{H}, \quad \delta>0, \quad x \in \operatorname{Im} Q_{1} .
$$

For the coefficients $b$ and $g$, we assume the following conditions. 


\section{Hypothesis 2.}

1. The mappings $b: \overline{\mathcal{O}} \times \mathbb{R} \rightarrow \mathbb{R}$ and $g: \overline{\mathcal{O}} \times \mathbb{R} \rightarrow \mathbb{R}$ are of class $C^{\infty}$ and have uniformly bounded derivatives.

For any $\xi \in \mathcal{O}, b(\xi, 0)=0$ and

$$
\sup _{\substack{\xi \in \mathcal{O} \\ \sigma \in \mathbb{R}}}|g(\xi, \sigma)|=: g_{1}<\infty .
$$

Moreover, $D_{\sigma} b(\xi, 0)$ and $g(\xi, 0)$ do not depend on $\xi \in \mathcal{O}$.

2. It holds that

$$
\sup _{\xi \in \mathcal{O}} \sup _{\substack{\sigma_{1}, \sigma_{2} \in \mathbb{R} \\ \sigma_{1} \neq \sigma_{2}}} \frac{\left|b\left(\xi, \sigma_{1}\right)-b_{2}\left(\xi, \sigma_{2}\right)\right|}{\left|\sigma_{1}-\sigma_{2}\right|}=: L_{b}<\lambda .
$$

3. We have

$$
\inf _{\substack{\xi \in \mathcal{O} \\ \sigma \in \mathbb{R}}}|g(\xi, \sigma)|=: g_{0}>0 .
$$

4. If $\beta$ is the constant introduced in (2.9), then for any $\gamma \leq \beta+\frac{1}{2}$

$$
\begin{aligned}
& x \in H_{\mathcal{N}_{\gamma}}^{2 \gamma, 2}(\mathcal{O}) \Rightarrow \mathcal{N}_{\gamma} b(\cdot, x)_{\left.\right|_{\partial \mathcal{O}}}=0 \\
& x, y \in H_{\mathcal{N}_{\gamma}}^{2 \gamma, 2}(\mathcal{O}) \Rightarrow \mathcal{N}_{\gamma}(g(\cdot, x) y)_{\left.\right|_{\partial \mathcal{O}}}=0
\end{aligned}
$$

and

$$
\begin{aligned}
& x, y \in H_{\mathcal{N}_{\beta}}^{2 \beta, 2}(\mathcal{O}) \Rightarrow \mathcal{N}_{\beta}\left(D_{\sigma} b(\cdot, x) y\right)_{\left.\right|_{\partial \mathcal{O}}}=0, \\
& x, y, z \in H_{\mathcal{N}_{\beta}}^{2 \beta, 2}(\mathcal{O}) \Rightarrow \mathcal{N}_{\beta}\left(D_{\sigma} g(\cdot, x) y z\right)_{\left.\right|_{\partial \mathcal{O}}}=0 .
\end{aligned}
$$

\section{Remark 2.2.}

1. In Hypothesis 2 we are assuming $b$ and $g$ to be of class $C^{\infty}$ for the sake of simplicity. In fact, it would be sufficient to assume that $b$ and $g$ are in $C^{k}(\overline{\mathcal{O}} \times \mathbb{R})$, for some $k$ large enough, depending on the constant $\beta$ introduced in (2.9) and on the order of the boundary operator $\mathcal{N}$.

2. The condition that both $D_{\sigma} b(\xi, 0)$ and $g(\xi, 0)$ do not depend on $\xi \in \mathcal{O}$ is assumed as in [5] in order to get local exact controllability for system (1.4) and hence a good description of the quasi-potential associated with (1.4).

3. Due to (2.23), we also have that the mapping

$$
(\xi, \sigma) \in \overline{\mathcal{O}} \times \mathbb{R} \rightarrow \frac{1}{g(\xi, \sigma)} \in \mathbb{R}
$$

is of class $C^{\infty}$, with uniformly bounded derivatives.

4. Condition 4 in Hypothesis 2 is satisfied once we impose suitable conditions on $b, g$ and their derivatives. For example, in the case $\mathcal{N}=I$, we have that (2.24) is satisfied if

$$
D_{\sigma}^{j} b(\xi, 0)=D_{\sigma}^{j} g(\xi, 0)=0, \quad \xi \in \partial \mathcal{O},
$$

for any $j=1, \ldots, 2 k$, where $k \in[\beta-5 / 4, \beta-1 / 4]$. Analogously, condition (2.25) is satisfied if the same condition for the derivatives of $b$ and $g$ are satisfied up to the order $2 k+1$. 
In what follows, for any $x, y \in H$ we shall denote

$$
B(x)(\xi):=b(\xi, x(\xi)), \quad \xi \in \mathcal{O},
$$

and

$$
[G(x) y](\xi):=g(\xi, x(\xi)) y(\xi), \quad \xi \in \mathcal{O} \text {. }
$$

Due to our assumptions, the mapping $B: H \rightarrow H$ is well defined and Gâteaux differentiable, with

$$
\left[B^{\prime}(x) y\right](\xi)=D_{\sigma} b(\xi, x(\xi)) y(\xi), \quad \xi \in \mathcal{O} .
$$

In particular, $B^{\prime}$ is bounded, so that $B: H \rightarrow H$ is Lipschitz continuous.

If we take $x \in H^{2 \gamma, 2}(\mathcal{O}) \cap L^{\infty}(\mathcal{O})$, then, as proved in [12, Theorem 5.5.4.1], we have that $B(x) \in H^{2 \gamma, 2}(\mathcal{O})$ and

$$
|B(x)|_{H^{2 \gamma, 2}(\mathcal{O})} \leq c|x|_{H^{2 \gamma, 2}(\mathcal{O})}\left(1+|x|_{L^{\infty}(\mathcal{O})}^{r}\right),
$$

for some $r \geq 1$. Moreover, according to (2.24), we have that if $x \in H_{\mathcal{N}_{\gamma}}^{2 \gamma, 2}(\mathcal{O})$, then $B(x) \in H_{\mathcal{N}_{\gamma}}^{2 \gamma, 2}(\mathcal{O})$. Thanks to (2.4), this implies

$$
x \in D\left((-A)^{\gamma}\right) \cap L^{\infty}(\mathcal{O}) \Longrightarrow B(x) \in D\left((-A)^{\gamma}\right)
$$

and

$$
|B(x)|_{D\left((-A)^{\gamma}\right)} \leq c|x|_{D\left((-A)^{\gamma}\right)}\left(1+|x|_{L^{\infty}(\mathcal{O})}^{r}\right) .
$$

Concerning the mapping $G$, due to (2.21) we have that $G: H \rightarrow \mathcal{L}(H)$ is well defined and bounded, but, in spite of the fact that $D_{\sigma} g$ is uniformly bounded, $G$ : $H \rightarrow \mathcal{L}(H)$ is not Lipschitz continuous. We only have that $G: H \rightarrow \mathcal{L}\left(H, L^{1}(\mathcal{O})\right)$ and $G: H \rightarrow \mathcal{L}\left(L^{\infty}(\mathcal{O}), H\right)$ are Lipschitz continuous.

Moreover, as (2.23) holds, $G(x)$ is invertible as an operator in $H$, for any $x \in H$, and for any $x, y \in H$,

$$
\left[G^{-1}(x) y\right](\xi)=\frac{y(\xi)}{g(\xi, x(\xi))}, \quad \xi \in \mathcal{O} .
$$

Notice that $G^{-1}: H \rightarrow \mathcal{L}(H)$ is well defined and bounded. Moreover, from (2.23) and (2.24) together, we have that for any $\gamma \leq \beta+\frac{1}{2}$

$$
x, y \in H_{\mathcal{N}_{\gamma}}^{2 \gamma, 2}(\mathcal{O}) \Rightarrow \mathcal{N}_{\gamma}\left(g(\cdot, x)^{-1} y\right)_{\left.\right|_{\partial \mathcal{O}}}=0 .
$$

According to Hypotheses 1 and 2 , for any $x \in H$ and $\epsilon, \delta>0$ the problem

$$
\left\{\begin{array}{l}
\frac{\partial u}{\partial t}(t, \xi)=\mathcal{A} u(t, \xi)+b(\xi, u(t, \xi))+\sqrt{\epsilon} g(\xi, u(t, \xi)) \frac{\partial w^{Q_{\delta}}}{\partial t}(t, \xi), \\
u(0, \xi)=x(\xi), \quad \mathcal{N} u(t, \xi)=0, \quad \xi \in \partial \mathcal{O}
\end{array}\right.
$$

admits a unique mild solution $u_{\epsilon, \delta}^{x} \in L^{p}(\Omega ; C([0, T] ; H))$, for any $T>0$ and $p \geq 1$. Moreover, as proved in [3], there exists some $\epsilon_{0}>0$ and $\gamma_{1}>0$ such that for any $\epsilon \leq \epsilon_{0}$ and $p \geq 1$,

$$
\mathbb{E}\left|u_{\epsilon, \delta}^{x}(t)\right|_{H}^{p} \leq c_{p, \delta}\left(1+e^{-\gamma_{1} p t}|x|_{H}^{p}\right), \quad t \geq 0 .
$$

Moreover, as shown in [3], there exists some $\theta>0$ such that for any $a>0$,

$$
\sup _{t \geq a} \mathbb{E}\left|u_{\epsilon, \delta}^{x}(t)\right|_{D\left((-A)^{\theta}\right)} \leq c_{a, \delta}\left(1+|x|_{H}\right) .
$$


Now, for any $\epsilon, \delta>0$ we denote by $P_{t}^{\epsilon, \delta}$ the transition semigroup associated with problem (2.30), which is defined by

$$
P_{t}^{\epsilon, \delta} \varphi(x)=\mathbb{E} \varphi\left(u_{\epsilon, \delta}^{x}(t)\right), \quad t \geq 0, \quad x \in H,
$$

for any $\varphi \in B_{b}(H)=\{\varphi: H \rightarrow \mathbb{R}$, bounded and Borel $\}$. Due to (2.32), for any $\epsilon<\epsilon_{0}$ the family $\left\{\mathcal{L}\left(u_{\epsilon, \delta}^{x}(t)\right)\right\}_{t \geq a}$ is tight in $\mathcal{P}(H, \mathcal{B}(H))$, and then by the KrylovBogoliubov theorem, the family of measures $\left\{\nu_{\epsilon, \delta}^{T}\right\}_{T>0}$ defined by

$$
\nu_{\epsilon, \delta}^{T}(A):=\frac{1}{T} \int_{0}^{T} P_{t}^{\epsilon} \mathbb{I}_{A}(0) d t, \quad T>0
$$

is tight in $\mathcal{P}(H)$ and the weak limit $\nu_{\epsilon, \delta}$, as $T \uparrow+\infty$, is invariant for the semigroup $P_{t}^{\epsilon, \delta}$.

As in [3, Theorem 7.4], it is possible to show that if $\epsilon_{0}$ is small enough, there exists $\gamma_{2}>0$ such that

$$
\mathbb{E}\left|u_{\epsilon, \delta}^{x}(t)-u_{\epsilon, \delta}^{y}(t)\right|_{H} \leq c_{\delta} e^{-\gamma_{2} t}|x-y|_{H}, \quad t \geq 0,
$$

for any $\epsilon \leq \epsilon_{0}$ and $x, y \in H$. In particular, this implies that for any $\epsilon$ sufficiently small $\nu_{\epsilon, \delta}$ is the unique invariant measure for $P_{t}^{\epsilon, \delta}$ and is strongly mixing.

\section{Some Basic FaCts on RELAXATION AND $\Gamma$-CONVERGENCE}

For the reader's convenience, we recall some definitions and results on the notion of relaxation of functions and of $\Gamma$-convergence for sequences of functionals (for a reference see e.g. the monograph $[\underline{8}$ ).

In the present section we shall denote by $X$ a topological space which satisfies the first axiom of countability, and for any $x \in X$ we shall denote by $\mathcal{N}(x)$ the set of all open neighborhoods of $x$ in $X$.

Definition 3.1. Let $F: X \rightarrow \overline{\mathbb{R}}$ be a function.

1. $F$ is lower semi-continuous if the set $\{F \leq t\}$ is closed in $X$, for any $t \in \mathbb{R}$.

2. $F$ is coercive if the closure of the set $\{F \leq t\}$ is countably compact, for any $t \in \mathbb{R}$.

Now, let $\left\{F_{n}\right\}_{\in \mathbb{N}}$ be a sequence of functions all defined in $X$ with values in $\overline{\mathbb{R}}$.

Definition 3.2. The sequence $\left\{F_{n}\right\} \in \mathbb{N}$ is equi-coercive if for any $t \in \mathbb{R}$ there exists a closed countably compact set $K_{t} \subset X$ such that

$$
\left\{F_{n} \leq t\right\} \subseteq K_{t}, \quad n \in \mathbb{N} .
$$

As proved in [8, Proposition 7.7], the following characterization of equi-coercive sequences holds.

Proposition 3.3. The sequence $\left\{F_{n}\right\} \in \mathbb{N}$ is equi-coercive if and only if there exists a lower semi-continuous coercive function $\Psi: X \rightarrow \overline{\mathbb{R}}$ such that

$$
F_{n}(x) \geq \Psi(x), \quad x \in X, \quad n \in \mathbb{N} .
$$

Now, we introduce the notion of relaxation for a function $F$.

Definition 3.4. The lower semi-continuous envelope, or relaxed function, of a function $F: X \rightarrow \overline{\mathbb{R}}$ is defined by

$$
\left(s c^{-} F\right)(x)=\sup _{G \in \mathcal{G}(F)} G(x), \quad x \in X,
$$

where $\mathcal{G}(F)$ is the set of all lower semi-continuous functions $G \leq F$. 
From the definition, one immediately has that $s c^{-} F$ is lower semi-continuous, $s c^{-} F \leq F$ and $s c^{-} F \geq G$, for any $G \in \mathcal{G}(F)$, so that $s c^{-} F$ can be regarded as the greatest lower semi-continuous function majorized by $F$. Moreover, it is possible to prove that

$$
\left(s c^{-} F\right)(x)=\sup _{U \in \mathcal{N}(x)} \inf _{y \in U} F(y), \quad x \in X
$$

(see [8, Proposition 3.3]).

The following result, whose proof can be found in [8, Proposition 3.6], provides a possible characterization of $s c^{-} F$ which we will use later on in this paper.

Proposition 3.5. For any function $F: X \rightarrow \overline{\mathbb{R}}$, its lower semi-continuous function $s c^{-} F$ is characterized by the following properties:

1. for any $x \in X$ and any sequence $\left\{x_{n}\right\}_{n \in \mathbb{N}}$ converging to $x$ in $X$, it holds that

$$
\left(s c^{-} F\right)(x) \leq \liminf _{n \rightarrow \infty} F\left(x_{n}\right)
$$

2. for any $x \in X$ there exists a sequence $\left\{x_{n}\right\}_{n \in \mathbb{N}}$ converging to $x$ in $X$ such that

$$
\left(s c^{-} F\right)(x) \geq \limsup _{n \rightarrow \infty} F\left(x_{n}\right)
$$

Next, we introduce the notion of $\Gamma$-convergence for sequences of functions.

Definition 3.6. The $\Gamma$-lower limit and the $\Gamma$-upper limit of the sequence $\left\{F_{n}\right\}_{n \in \mathbb{N}}$ are the functions from $X$ into $\overline{\mathbb{R}}$ defined respectively by

$$
\begin{aligned}
& \Gamma-\liminf _{n \rightarrow \infty} F_{n}(x)=\sup _{U \in \mathcal{N}(x)} \liminf _{n \rightarrow \infty} \inf _{y \in U} F_{n}(y), \\
& \Gamma-\limsup _{n \rightarrow \infty} F_{n}(x)=\sup _{U \in \mathcal{N}(x)} \limsup _{n \rightarrow \infty} \inf _{y \in U} F_{n}(y) .
\end{aligned}
$$

If there exists a function $F: X \rightarrow \overline{\mathbb{R}}$ such that $\Gamma-\lim \inf _{n \rightarrow \infty} F_{n}=\Gamma-\lim \sup _{n \rightarrow \infty} F_{n}$ $=F$, then we write

$$
F=\Gamma-\lim _{n \rightarrow \infty} F_{n}
$$

and we say that the sequence $\left\{F_{n}\right\}_{n \in \mathbb{N}}$ is $\Gamma$-convergent to $F$.

In [8, Proposition 5.7] we can find the proof of the following result, which links $\Gamma$-convergence and relaxation of functions and provides a useful criterium for $\Gamma$ convergence.

Proposition 3.7. If $\left\{F_{n}\right\}_{n \in \mathbb{N}}$ is a decreasing sequence converging to $F$ pointwise, then $\left\{F_{n}\right\}_{n \in \mathbb{N}}$ is $\Gamma$-convergent to $s c^{-} F$.

We conclude by giving a criterium for convergence of minima for $\Gamma$-convergent sequences (for a proof see [8, Theorem 7.8]).

Theorem 3.8. Suppose that the sequence $\left\{F_{n}\right\}_{n \in \mathbb{N}}$ is equi-coercive in $X$ and $\Gamma$ converges to a function $F$ in $X$. Then, $F$ is coercive and

$$
\min _{x \in X} F(x)=\lim _{n \rightarrow \infty} \inf _{x \in X} F_{n}(x) .
$$




\section{The SKeleton EQUATion}

We first consider the unperturbed system

$$
\left\{\begin{array}{l}
\frac{\partial u}{\partial t}(t, \xi)=\mathcal{A} u(t, \xi)+b(\xi, u(t, \xi)), \quad t \geq s, \\
u(s, \xi)=x(\xi), \quad \mathcal{N} u(t, \xi)=0, \quad \xi \in \partial \mathcal{O},
\end{array}\right.
$$

which can be written as the following abstract evolution equation in the Hilbert space $H$ :

$$
u^{\prime}(t)=A u(t)+B(u(t)), \quad u(s)=x .
$$

In what follows we shall denote its solution by $u^{x}(t ; s)$ and sometimes, when $s=0$, we shall write $u^{x}(t)$. Due to our assumptions on the diffusion operator $A$ and the reaction term $B$, we have the following fact (this is a known fact, but we give a proof for completeness).

Proposition 4.1. Assume that $b(\xi, \cdot): \mathbb{R} \rightarrow \mathbb{R}$ is Lipschitz continuous, with $b(\xi, 0)=0$, and assume that (2.22) holds. Then, for any $\gamma \leq 1 / 2, x \in D\left((-A)^{\gamma}\right)$ and $s \geq 0$ the solution $u^{x}(\cdot ; s)$ of problem (4.1) belongs to $L^{\infty}\left((s,+\infty) ; D\left((-A)^{\gamma}\right)\right)$ and

$$
\left|u^{x}(t ; s)\right|_{D((-A) \gamma)} \leq e^{-\left(\lambda-L_{b}\right)(t-s)}|x|_{D((-A) \gamma)}, \quad t \geq s .
$$

Proof. First of all notice that for any $\gamma_{1}<\gamma_{2}$ and $x \in D\left((-A)^{\gamma_{2}}\right)$,

$$
|x|_{D\left((-A)^{\gamma_{1}}\right)} \leq \lambda^{-\left(\gamma_{2}-\gamma_{1}\right)}|x|_{D\left((-A)^{\gamma_{2}}\right)} .
$$

For any $\epsilon>0$ we have

$$
\begin{aligned}
& \frac{1}{2} \frac{d}{d t}\left|(-A)^{\gamma} u^{x}(t ; s)\right|_{H}^{2}=-\left|(-A)^{\frac{2 \gamma+1}{2}} u^{x}(t ; s)\right|_{H}^{2}+\left\langle(-A)^{2 \gamma} u^{x}(t ; s), B\left(u^{x}(t ; s)\right)\right\rangle_{H} \\
& \leq-\left|(-A)^{\frac{2 \gamma+1}{2}} u^{x}(t ; s)\right|_{H}^{2}+\frac{\epsilon}{2}\left|(-A)^{2 \gamma} u^{x}(t ; s)\right|_{H}^{2}+\frac{1}{2 \epsilon}\left|B\left(u^{x}(t ; s)\right)\right|_{H}^{2} \\
& \leq-\left|(-A)^{\frac{2 \gamma+1}{2}} u^{x}(t ; s)\right|_{H}^{2}+\frac{\epsilon}{2}\left|(-A)^{2 \gamma} u^{x}(t ; s)\right|_{H}^{2}+\frac{L_{b}^{2}}{2 \epsilon}\left|u^{x}(t ; s)\right|_{H}^{2} .
\end{aligned}
$$

Thus, according to (4.3), as we are assuming $\gamma \leq 1 / 2$, we have

$$
\begin{aligned}
& \frac{1}{2} \frac{d}{d t}\left|(-A)^{\gamma} u^{x}(t ; s)\right|_{H}^{2} \leq-\left(1-\frac{\epsilon}{2 \lambda^{1-2 \gamma}}\right)\left|(-A)^{\frac{2 \gamma+1}{2}} u^{x}(t ; s)\right|_{H}^{2}+\frac{L_{b}^{2}}{2 \epsilon}\left|u^{x}(t ; s)\right|_{H}^{2} \\
& \leq-\left(\lambda-\frac{\epsilon \lambda^{2 \gamma}}{2}-\frac{L_{b}^{2}}{2 \epsilon \lambda^{2 \gamma}}\right)\left|(-A)^{\gamma} u^{x}(t ; s)\right|_{H}^{2} .
\end{aligned}
$$

Now, if we take $\epsilon=L_{b} \lambda^{-2 \gamma}$, we obtain

$$
\frac{1}{2} \frac{d}{d t}\left|(-A)^{\gamma} u^{x}(t ; s)\right|_{H}^{2} \leq-\left(\lambda-L_{b}\right)\left|(-A)^{\gamma} u^{x}(t ; s)\right|_{H}^{2},
$$

and this implies (4.2).

Notice that an estimate analogous to (4.2) also holds if the $D\left((-A)^{\gamma}\right)$-norm is replaced by the $L^{\infty}(\mathcal{O})$-norm, that is, $u^{x}(\cdot ; s) \in L^{\infty}\left((s,+\infty) ; L^{\infty}(\mathcal{O})\right)$, for any $x \in L^{\infty}(\mathcal{O})$, and

$$
\left|u^{x}(t ; s)\right|_{L^{\infty}(\mathcal{O})} \leq e^{-\left(\lambda-L_{b}\right)(t-s)}|x|_{L^{\infty}(\mathcal{O})}, \quad t \geq s .
$$


Now, for any $c>0$ and $\gamma \geq 0$ we define

$$
B_{c, \gamma}=\left\{x \in D\left((-A)^{\gamma}\right):|x|_{D\left((-A)^{\gamma}\right)} \leq c\right\},
$$

and for any $c>0$

$$
B_{c, \infty}=\left\{x \in L^{\infty}(\mathcal{O}):|x|_{L^{\infty}(\mathcal{O})} \leq c\right\} .
$$

Moreover, for any $x \in H, \gamma \geq 0$ and $c>0$ we define

$$
t_{c, \gamma}^{x}:=\inf \left\{t \geq 0: u^{x}(t ; 0) \in B_{c, \gamma} \cap B_{c, \infty}\right\} .
$$

As a consequence of (4.2) and (4.4), we have the following fact.

Lemma 4.2. For any $\gamma \geq 0$ and $c_{1}, c_{2}>0$ there exists $T=T\left(\gamma, d, c_{1}, c_{2}\right)>0$ such that

$$
\sup _{x \in B_{c_{1}, 0}} t_{c_{2}, \gamma}^{x}<T
$$

and

$$
u^{x}(t ; 0) \in B_{c_{2}, \gamma} \cap B_{c_{2}, \infty}, \quad t>T, \quad x \in B_{c_{1}, 0} .
$$

Proof. If we show that there exists some $c_{d}>0$ such that for any $k \in \mathbb{N} \cup\{0\}$ and $x \in H$

$$
d \in[4 k, 4(k+1)) \Longrightarrow\left|u^{x}(t ; 0)\right|_{L^{\infty}(\mathcal{O})} \leq c_{d}|x|_{H}, \quad t \geq k+1,
$$

then, according to (4.4), we get

$$
\begin{aligned}
& \left|u^{x}(t ; 0)\right|_{L^{\infty}(\mathcal{O})}=\left|u^{u^{x}(k+1 ; 0)}(t ; k+1)\right|_{L^{\infty}(\mathcal{O})} \\
& \leq e^{-\left(\lambda-L_{b}\right)(t-k-1)}\left|u^{x}(k+1 ; 0)\right|_{L^{\infty}(\mathcal{O})} \leq c_{d} e^{-\left(\lambda-L_{b}\right)(t-k-1)}|x|_{H}, \quad t \geq k+1 .
\end{aligned}
$$

Hence we can fix $T_{1}=T_{1}\left(d, c_{1}, c_{2}\right)>0$ such that

$$
x \in B_{c_{1}, 0} \Longrightarrow\left|u^{x}(t ; 0)\right|_{L^{\infty}(\mathcal{O})} \leq c_{2}, \quad t \geq T_{1} .
$$

Next, if we show that for any $s \geq 0$ and $\gamma \in((h-1) / 4, h / 4]$, with $h \in \mathbb{N}$, there exists $\kappa_{\gamma}$ such that

$$
\left|u^{x}(t ; s)\right|_{D\left((-A)^{\gamma}\right)} \leq c_{\gamma}|x|_{H}^{\kappa_{\gamma}}, \quad t-s \geq t_{h}:=h \vee(k+2),
$$

where $k$ is defined by (4.5), then for $t \geq s+t_{h}$ we have

$$
\left|u^{x}(t ; 0)\right|_{D\left((-A)^{\gamma}\right)}=\left|u^{u^{x}(s ; 0)}(t ; s)\right|_{D\left((-A)^{\gamma}\right)} \leq c_{\gamma}\left|u^{x}(s ; 0)\right|_{H}^{\kappa_{\gamma}} .
$$

According to (4.2), we get

$$
\left|u^{x}(t ; 0)\right|_{D\left((-A)^{\gamma}\right)} \leq c_{\gamma} e^{-\kappa_{\gamma}\left(\lambda-L_{b}\right) s}|x|_{H}^{\kappa_{\gamma}}, \quad t \geq s+t_{h},
$$

and this implies that there exists $T_{2}=T_{2}\left(\gamma, d, c_{1}, c_{2}\right)>0$ such that

$$
x \in B_{c_{1}, 0} \Longrightarrow\left|u^{x}(t ; 0)\right|_{D\left((-A)^{\gamma}\right)} \leq c_{2}, \quad t>T_{2} .
$$

Together with (4.6), this implies the lemma, once we set

$$
T\left(\gamma, d, c_{1}, c_{2}\right):=T_{1}\left(\delta, c_{1}, c_{2}\right) \vee T_{2}\left(\gamma, d, c_{1}, c_{2}\right) .
$$

Therefore, it remains to prove (4.5) and (4.7). 
Step 1, proof of (4.5). If $k=0$ (that is, $d<4$ ), then, due to (2.5) and (4.2), we have

$$
\left|u^{x}(t ; 0)\right|_{L^{\infty}(\mathcal{O})} \leq c t^{-\frac{d}{4}}|x|_{H}+c \int_{0}^{t} e^{-\lambda(t-s)}(t-s)^{-\frac{d}{4}}\left|u^{x}(s ; 0)\right|_{H} d s \leq c\left(t^{-\frac{d}{4}}+1\right)|x|_{H},
$$

so that (4.5) holds for $t \geq 1$. Now, assume that $d \in[4 k, 4(k+1))$, for $k \geq 1$. It is immediate to check that we can fix $q_{1}<\cdots<q_{k}$ such that

$$
2<q_{1}<\frac{2 d}{d-4}, \quad q_{k}>\frac{d}{2}, \quad q_{i}>\frac{d q_{i+1}}{2 q_{i+1}+d}, i=1, \ldots, k-1 .
$$

Then, by an inductive argument, it is possible to show that

$$
\left|u^{x}(t ; 0)\right|_{L^{q_{i}}(\mathcal{O})} \leq c_{i}|x|_{H}, \quad t \geq i, \quad i=1, \ldots, k .
$$

Actually, for $i=1$, we have

$$
\left|u^{x}(t ; 0)\right|_{L^{q_{1}}(\mathcal{O})} \leq c t^{-\frac{d\left(q_{1}-2\right)}{4 q_{1}}}|x|_{H}+c \int_{0}^{t} e^{-\lambda(t-s)}(t-s)^{-\frac{d\left(q_{1}-2\right)}{4 q_{1}}}\left|u^{x}(s ; 0)\right|_{H} d s,
$$

and then, as $q_{1}<2 d /(d-4)$, from (4.2) we get (4.8). Now, if (4.8) is valid for some $i \geq 1$, then for any $t>i$ we have

$$
\begin{aligned}
& \left|u^{x}(t ; 0)\right|_{L^{q_{i+1}}(\mathcal{O})} \leq c(t-i)^{-\frac{d\left(q_{i+1}-q_{i}\right)}{2 q_{i} q_{i+1}}}\left|u^{x}(i ; 0)\right|_{L^{q_{i}}(\mathcal{O})} \\
& +c \int_{i}^{t} e^{-\lambda(t-s)}(t-s)^{-\frac{d\left(q_{i+1}-q_{i}\right)}{2 q_{i} q_{i+1}}}\left|u^{x}(s ; 0)\right|_{L^{q_{i}}(\mathcal{O})} d s \leq c_{i}\left((t-i)^{-\frac{d\left(q_{i+1}-q_{i}\right)}{2 q_{i} q_{i+1}}}+1\right)|x|_{H},
\end{aligned}
$$

so that (4.8) is also true for $i+1$.

Now, in view of (4.8), we have

$$
\left|u^{x}(t ; 0)\right|_{L^{q_{k}(\mathcal{O})}} \leq c_{k}|x|_{H}, \quad t \geq k,
$$

so that, as $q_{k}>d / 2$, for $t \geq k$ we get

$$
\begin{aligned}
\left|u^{x}(t ; 0)\right|_{L^{\infty}(\mathcal{O})} \leq c & (t-k)^{-\frac{d}{2 q_{k}}}\left|u^{x}(k ; 0)\right|_{L^{q_{k}}(\mathcal{O})} \\
& +c \int_{k}^{t} e^{-\lambda(t-s)}(t-s)^{-\frac{d}{2 q_{k}}}\left|u^{x}(s ; 0)\right|_{L^{q_{k}}(\mathcal{O})} d s
\end{aligned}
$$

which implies (4.5).

Step 2, proof of (4.7). Thanks to (2.5) it is immediate to check that for any $h \in$ $L^{\infty}\left(\left(T_{1}, T_{2}\right) ; D\left((-A)^{\alpha}\right)\right)$, with $\alpha \geq 0$ and $T_{1}<T_{2}$, it holds that

$$
\left|\int_{T_{1}}^{T_{2}} e^{\left(T_{2}-s\right) A} h(s) d s\right|_{D\left((-A)^{\left.\alpha+\frac{1}{4}\right)}\right.} \leq c_{\alpha}|h|_{L^{\infty}\left(\left(T_{1}, T_{2}\right) ; D\left((-A)^{\alpha}\right)\right)}
$$

for some constant $c_{\alpha}$ independent of $T_{1}$ and $T_{2}$. Then, from (2.5), (4.2) and (4.9) we have

$$
\left|u^{x}(t ; 0)\right|_{D\left((-A)^{\left.\frac{1}{4}\right)}\right.} \leq c t^{-\frac{1}{4}}|x|_{H}+c\left|u^{x}(\cdot ; 0)\right|_{L^{\infty}\left(\left(T_{1}, T_{2}\right) ; H\right)} \leq c\left(t^{-\frac{1}{4}}+1\right)|x|_{H},
$$

so that

$$
\left|u^{x}(t ; 0)\right|_{D\left((-A)^{\frac{1}{4}}\right)} \leq 2 c|x|_{H}, \quad t \geq 1 .
$$


If $\gamma \leq 1 / 4$ this concludes the proof of (4.7). Thus, assume that $\gamma>1 / 4$; that is, $h>1$. Thanks to (2.27), in view of (4.5) and (4.10) we have that $B\left(u^{x}(t ; 0)\right) \in$ $D\left((-A)^{\frac{1}{4}}\right)$, for any $t \geq t_{1}$, and

$$
\begin{array}{r}
\left|B\left(u^{x}(t ; 0)\right)\right|_{D\left((-A)^{\frac{1}{4}}\right)} \leq c\left|u^{x}(t ; 0)\right|_{D\left((-A)^{\frac{1}{4}}\right)}\left(1+\left|u^{x}(t ; 0)\right|_{L^{\infty}(\mathcal{O})}^{r}\right) \leq c\left(1+|x|_{H}^{r+1}\right), \\
t \geq t_{1} .
\end{array}
$$

Hence, by using (4.9) once more, we conclude that

$$
\left|u^{x}(t ; 0)\right|_{D\left((-A)^{\frac{1}{2}}\right)} \leq c\left(t-t_{1}\right)^{-\frac{1}{4}}\left|u^{x}\left(t_{1} ; 0\right)\right|_{D\left((-A)^{\frac{1}{4}}\right)}+c\left(1+|x|_{H}^{r+1}\right), \quad t \geq t_{1},
$$

so that

$$
\left|u^{x}(t ; 0)\right|_{D\left((-A)^{\frac{1}{2}}\right)} \leq c\left(1+|x|_{H}^{r+1}\right), \quad t \geq t_{1}+1 .
$$

By proceeding in this way, we get (4.7).

Next, for any $-\infty \leq t_{1}<t_{2} \leq+\infty$ and $\varphi \in L^{2}\left(\left(t_{1}, t_{2}\right) ; H\right)$, we denote by $z(\varphi)$ the mild solution in $C\left(\left[t_{1}, t_{2}\right] ; H\right)$ of the problem

$$
z^{\prime}(t)=A z(t)+B(z(t))+G(z(t)) \varphi(t), \quad z\left(t_{1}\right)=x \in H .
$$

When we need to emphasize that $z(\varphi)$ starts from $x$ at time $t_{1}$, we shall denote $z(\varphi)$ by $z_{t_{1}}^{x}(\varphi)$. Notice that in general the mapping $x \in H \mapsto G(x) y \in H$ is not Lipschitz continuous, for fixed $y \in H$. Then the existence and the uniqueness of solutions for problem (4.11) is not a trivial issue.

In the next proposition we give some estimates for $z(\varphi)$. An analogous result has also been proved in [5. Theorem 3.1], under a different setting of hypotheses on the coefficients.

Proposition 4.3. Under Hypotheses 1 and 2 , for any $\alpha \leq 1 / 2$ there exists $c_{\alpha}>0$ such that for any $T>0$ and $x \in H$

$$
\begin{array}{r}
\left|z_{-T}^{x}(\varphi)(t)\right|_{D\left((-A)^{\alpha}\right)} \leq c_{\alpha}\left[1+|\varphi|_{L^{2}((-T, 0) ; H)}+|x|_{H}\left(1+(t+T)^{-\alpha}\right)\right], \\
t \in(-T, 0] .
\end{array}
$$

Proof. For any $\varphi \in L^{2}((-\infty, 0) ; H), u \in C((-\infty, 0] ; H)$ and $T>0$ we define

$$
\gamma_{\varphi}^{T}(u)(t):=\int_{-T}^{t} e^{(t-s) A} G(u(s)) \varphi(s) d s, \quad-T \leq t \leq 0 .
$$

Due to the boundedness condition (2.21) on $g$ we have $|G(u(s)) \varphi(s)|_{H} \leq c|\varphi(s)|_{H}$. Then, in view of (2.8), for any $T>0$ and $s>-T$ and for any $\alpha \leq 1 / 2$ we obtain

$$
\sup _{t \in[-T, s]}\left|\gamma_{\varphi}^{T}(u)(t)\right|_{D\left((-A)^{\alpha}\right)} \leq c_{\alpha}|\varphi|_{L^{2}((-T, s) ; H)},
$$

with $c_{\alpha}$ independent of $T$ and $s$. Now, if we set $\gamma(t):=\gamma_{\varphi}^{T}\left(z_{-T}^{x}(\varphi)\right)(t)$ and $u(t):=$ $z_{-T}^{x}(\varphi)(t)-\gamma(t)$, we have

$$
\begin{aligned}
& \frac{1}{2} \frac{d}{d t}|u(t)|_{H}^{2}=\langle A u(t), u(t)\rangle_{H}+\langle B(u(t)+\gamma(t))-B(\gamma(t)), u(t)\rangle_{H}+\langle B(\gamma(t)), u(t)\rangle_{H} \\
& \leq-\frac{\lambda-L_{b}}{2}|u(t)|_{H}^{2}+c\left(|\gamma(t)|_{H}^{2}+1\right),
\end{aligned}
$$


so that, by comparison,

$$
|u(t)|^{2} \leq|x|_{H}^{2}+c \int_{-T}^{t} e^{-\left(\lambda-L_{b}\right)(t-s)}\left(|\gamma(s)|_{H}^{2}+1\right) d s, \quad t \geq-T .
$$

Thanks to (4.13), this implies

$$
\begin{aligned}
& \left|z_{-T}^{x}(\varphi)(t)\right|_{H}^{2} \leq 2|\gamma(t)|_{H}^{2}+2|x|_{H}^{2}+c \int_{-T}^{t} e^{-\left(\lambda-L_{b}\right)(t-s)}\left(|\gamma(s)|_{H}^{2}+1\right) d s \\
& \leq 2|x|_{H}^{2}+c \sup _{s \in[-T, t]}|\gamma(s)|_{H}^{2} \leq 2|x|_{H}^{2}+c\left(|\varphi|_{L^{2}((-T, t) ; H)}^{2}+1\right),
\end{aligned}
$$

and this immediately yields (4.12) for $\alpha=0$.

Next, concerning (4.12) in the general case $\alpha \leq 1 / 2$, due to (2.8) and (4.14) we have

$$
\begin{aligned}
& \left|\int_{-T}^{t} e^{(t-s) A} B\left(z_{-T}^{x}(\varphi)(s)\right) d s\right|_{D\left((-A)^{\alpha}\right)} \leq c_{\alpha}\left|B\left(z_{-T}^{x}(\varphi)\right)\right|_{L^{2}((-T, t) ; H)} \\
& \leq c_{\alpha}\left|z_{-T}^{x}(\varphi)\right|_{L^{2}((-T, t) ; H)} \leq c_{\alpha}\left(1+|\varphi|_{L^{2}((-T, t) ; H)}+|x|_{H}\right) .
\end{aligned}
$$

Then, as

$$
z_{-T}^{x}(\varphi)(t)=e^{(t+T) A} x+\int_{-T}^{t} e^{(t-s) A} B\left(z_{-T}^{x}(\varphi)(s)\right) d s+\gamma_{\varphi}^{T}\left(z_{-T}^{x}(\varphi)\right)(t),
$$

from (4.13) and (4.15) it follows that

$$
\left|z_{-T}^{x}(\varphi)(t)\right|_{D\left((-A)^{\alpha}\right)} \leq c_{\alpha} e^{-\lambda(t+T)}((t+T) \wedge 1)^{-\alpha}|x|_{H}+\left(1+|\varphi|_{L^{2}((-T, t) ; H)}+|x|_{H}\right),
$$

which yields immediately (4.12).

Besides equation (4.11), for any $\delta>0$ we consider the problem

$$
z^{\prime}(t)=A z(t)+B(z(t))+G(z(t)) Q_{\delta} \varphi(t), \quad z\left(t_{1}\right)=x \in H .
$$

For any $t_{1}<t_{2}$ and $\varphi \in L^{2}\left(\left(t_{1}, t_{2}\right) ; H\right)$, equation (4.16) admits a unique mild solution in $C\left(\left[t_{1}, t_{2}\right] ; H\right)$, which we shall denote by $z_{t_{1}}^{\delta, x}(\varphi)$. Estimate (4.12) is also true for $z_{t_{1}}^{\delta, x}(\varphi)$, for a constant $c_{\alpha}$ which does not depend on $\delta>0$.

The following fact is proved in [5, Lemma 3.5] (in the case of continuous-valued solutions instead of $H$-valued solutions, as in the present case). The proof is heavily based on conditions (2.24) and (2.25) in Hypothesis 2.

Lemma 4.4. Let $z_{\delta}$ be the solution of the problem

$$
z_{\delta}(t)=\int_{-\infty}^{t} e^{(t-s) A} B\left(z_{\delta}(s)\right) d s+\int_{-\infty}^{t} e^{(t-s) A} G\left(z_{\delta}(s)\right) Q_{\delta} \varphi(s) d s,
$$

for some $\varphi \in L^{2}((-\infty, 0) ; H)$, and assume that

$$
\lim _{t \rightarrow-\infty}\left|z_{\delta}(t)\right|_{H}=0
$$

Then, under Hypotheses 1 and 2, we have that $z_{\delta} \in L^{\infty}\left((-\infty, 0) ; D\left((-A)^{\beta+\frac{1}{2}}\right)\right)$ and

$$
\lim _{t \rightarrow-\infty}\left|z_{\delta}(t)\right|_{D\left((-A)^{\beta+\frac{1}{2}}\right)}=0
$$


In an analogous way, it is possible to show that if

$$
z_{\delta}(t)=\int_{t_{0}}^{t} e^{(t-s) A} B\left(z_{\delta}(s)\right) d s+\int_{t_{0}}^{t} e^{(t-s) A} G\left(z_{\delta}(s)\right) Q_{\delta} \varphi(s) d s, \quad t \geq t_{0},
$$

for some $t_{0} \leq 0$ and $\varphi \in L^{2}\left(\left(t_{0}, 0\right) ; H\right)$, then

$$
z_{\delta} \in L^{\infty}\left(\left(t_{0}, 0\right) ; D\left((-A)^{\beta+\frac{1}{2}}\right)\right) .
$$

\section{Convergence of the APProximating ACtion-FUnCtionals}

We fix $x \in H$ once and for all, and we define

$$
\mathcal{X}_{x}:=\left\{u \in C((-\infty, 0) ; H): \lim _{t \rightarrow-\infty}|u(t)|_{H}=0, u(0)=x\right\} .
$$

The space $\mathcal{X}_{x}$ is endowed with the topology of uniform convergence on bounded intervals of $[-\infty, 0]$. Next, we denote

$$
\mathcal{Y}_{x}:=\left\{u \in \mathcal{X}_{x} \cap W_{2, \mathcal{N}}^{1,2}(-\infty, 0):\left|Q_{1}^{-1}(\mathcal{H}(u))\right|_{L^{2}((-\infty, 0) ; H)}<\infty\right\},
$$

where $Q_{1}^{-1}$ is the pseudo-inverse of the operator $Q_{1}$ introduced in equation (1.4), and corresponding to $\delta=1$,

$$
\mathcal{H}(u)(t, \xi)=\left(\partial_{t} u(t, \xi)-\mathcal{A} u(t, \xi)-b(\xi, u(t, \xi))\right)[g(\xi, u(t, \xi))]^{-1},
$$

and for any $-\infty \leq T_{1}<T_{2} \leq+\infty$

$$
\begin{array}{r}
W_{2, \mathcal{N}}^{1,2}\left(T_{1}, T_{2}\right):=\left\{u \in C\left(\left[T_{1}, T_{2}\right] ; H\right) \cap W_{2}^{1,2}\left(\left(T_{1}, T_{2}\right) \times \mathcal{O}\right): \mathcal{N} u(t)_{\left.\right|_{\partial \mathcal{O}}}=0,\right. \\
\left.t \in\left(T_{1}, T_{2}\right)\right\} .
\end{array}
$$

According to (2.15), for any $\delta>0$ we have

$$
\mathcal{Y}_{x}:=\left\{u \in \mathcal{X}_{x} \cap W_{2, \mathcal{N}}^{1,2}(-\infty, 0):\left|Q_{\delta}^{-1}(\mathcal{H}(u))\right|_{L^{2}((-\infty, 0) ; H)}<\infty\right\},
$$

so that on the space $\mathcal{X}_{x}$ we can define for any $\delta>0$ the operator

$$
S_{\delta}(u):= \begin{cases}\frac{1}{2}\left|Q_{\delta}^{-1}(\mathcal{H}(u))\right|_{L^{2}((-\infty, 0) ; H)}^{2}, & \text { if } u \in \mathcal{Y}_{x} \\ +\infty, & \text { otherwise }\end{cases}
$$

Moreover, on the space $\mathcal{X}_{x}$ we define the operators

$$
\tilde{S}(u):= \begin{cases}\frac{1}{2}|\mathcal{H}(u)|_{L^{2}((-\infty, 0) ; H)}^{2}, & \text { if } u \in \mathcal{Y}_{x} \\ +\infty, & \text { otherwise }\end{cases}
$$

and

$$
S(u):= \begin{cases}\frac{1}{2}|\mathcal{H}(u)|_{L^{2}((-\infty, 0) ; H)}^{2}, & \text { if } u \in \mathcal{X}_{x} \cap W_{2, \mathcal{N}}^{1,2}(-\infty, 0), \\ +\infty, & \text { otherwise. }\end{cases}
$$

Notice that

$$
\tilde{S}(u) \geq S(u), \quad u \in \mathcal{X}_{x}, \quad \tilde{S}(u)=S(u), \quad u \in \mathcal{Y}_{x} .
$$

Furthermore, if $d=1$, we have $Q_{\delta}=I$ for any $\delta>0$, and then $\mathcal{Y}_{x}=\mathcal{X}_{x} \cap$ $W_{2, \mathcal{N}}^{1,2}(-\infty, 0)$. This means in particular that $\tilde{S}(u)=S(u)$, for any $u \in \mathcal{X}_{x}$. 
Proposition 5.1. Assume Hypotheses 1 and 2. Then, the set $\mathcal{K}(r):=\{S(u) \leq r\}$ is compact in $\mathcal{X}_{x}$, for any $r \geq 0$. In particular, the functional $S: \mathcal{X}_{x} \rightarrow \overline{\mathbb{R}}$ is coercive and lower-semicontinuous.

Proof. We first notice that for any $\alpha \leq 1 / 2$ and for any $r \geq 0$,

$$
u \in \mathcal{K}(r) \Longrightarrow \sup _{t \leq 0}|u(t)|_{D\left((-A)^{\alpha}\right)} \leq L_{\alpha}(r)
$$

for some $L_{\alpha}(r) \geq 0$. Actually, if $u \in \mathcal{K}(r)$, then $S(u) \leq r$, so that

$$
|\mathcal{H}(u)|_{L^{2}((-\infty, 0) ; H)}^{2} \leq 2 r \text {. }
$$

Hence, with the same notation introduced in Section 4 , for any $T>0$ we have

$$
u(t)=z_{-T}^{u(-T)}(\mathcal{H}(u))(t), \quad t \in[-T, 0],
$$

and, according to (4.12), we obtain

$$
|u(t)|_{D\left((-A)^{\alpha}\right)} \leq c_{\alpha}\left[1+\sqrt{2 r}+|u(-T)|_{H}\left(1+(t+T)^{-\alpha}\right)\right], \quad t \in[-T, 0] .
$$

Now, since $u \in \mathcal{X}_{x}$, we have that $|u(-T)|_{H} \rightarrow 0$, as $T \rightarrow \infty$. Then, by taking the limit as $T$ goes to infinity in the right hand side of the inequality above, we conclude that

$$
|u(t)|_{D\left((-A)^{\alpha}\right)} \leq c_{\alpha}(1+\sqrt{2 r})=: L_{\alpha}(r), \quad t \leq 0 .
$$

Once we have (5.7), the proof of the compactness of $\mathcal{K}(r)$ proceeds exactly as in [13, proof of Proposition 6] and in [5, proof of Proposition 5.1].

Our aim in this section is proving that the family of functionals $\left\{S_{\delta}\right\}_{\delta>0}$ is $\Gamma$ convergent to the functional $S$ in $\mathcal{X}_{x}$. The first result going in that direction is the following one.

Proposition 5.2. Under Hypotheses 1 and 2, we have

$$
\Gamma-\lim _{\delta \rightarrow 0} S_{\delta}=s c^{-} \tilde{S}, \quad \text { in } \mathcal{X}_{x} .
$$

Proof. Thanks to (2.19) and to (2.18) (applied with $\delta_{1}=\delta$ and $\delta_{2}=1$ ), by using the dominate convergence theorem we get

$$
\lim _{\delta \rightarrow 0} S_{\delta}(u)=\tilde{S}(u), \quad u \in \mathcal{X}_{x} .
$$

In particular, due to (5.6), this implies that

$$
\lim _{\delta \rightarrow 0} S_{\delta}(u)=S(u), \quad u \in \mathcal{Y}_{x} .
$$

Moreover, thanks to (2.18) we have that

$$
\delta_{1}<\delta_{2} \Longrightarrow S_{\delta_{1}}(u) \leq S_{\delta_{2}}(u) .
$$

According to Proposition 3.7, this implies our thesis.

Once we have proved that $S_{\delta}$ is $\Gamma$-convergent to $s c^{-} \tilde{S}$ in $\mathcal{X}_{x}$, as $\delta \downarrow 0$, in order to prove that $S_{\delta}$ is $\Gamma$-convergent to $S$ it remains to prove that $s c^{-} \tilde{S}$ coincides with $S$. This is the most delicate step.

Theorem 5.3. Assume that $x \in D\left((-A)^{\beta+\frac{1}{2}}\right)$. Then, under Hypotheses 1 and 2 , we have that $\operatorname{sc}^{-} \tilde{S}(u)=S(u)$, for any $u \in \mathcal{X}_{x}$. In particular

$$
\Gamma-\lim _{\delta \rightarrow 0} S_{\delta}=S, \quad \text { in } \mathcal{X}_{x} .
$$


Proof. Notice that in dimension $d=1$ we have $\mathcal{Y}_{x}=\mathcal{X}_{x} \cap W_{2, \mathcal{N}}^{1,2}(-\infty, 0)$ and $\tilde{S}(u)=S(u)$, for any $u \in \mathcal{X}_{x}$. Thus, as $S$ is lower semi-continuous (see Proposition 5.1), we have $s c^{-} \tilde{S}=S$ and the theorem is proved. Now assume $d \geq 2$. In order to prove that $s c^{-} \tilde{S}(u)$ coincides with $S(u)$, for any $u \in \mathcal{X}_{x}$, we use the characterization of $s c^{-} \tilde{S}$ given in Proposition 3.5 .

Step 1 . We show that for any $u \in \mathcal{X}_{x}$ and any sequence $\left\{u_{n}\right\}_{n \in \mathbb{N}} \subset \mathcal{X}_{x}$ such that

$$
\lim _{n \rightarrow \infty} \sup _{t \leq 0}\left|u_{n}(t)-u(t)\right|_{H}=0
$$

it holds that

$$
S(u) \leq \liminf _{n \rightarrow \infty} \tilde{S}\left(u_{n}\right) .
$$

In Proposition 5.1 we have shown that the functional $S$ is lower-semicontinuous. Then

$$
S(u) \leq \liminf _{n \rightarrow \infty} S\left(u_{n}\right) .
$$

Due to (5.6), this allows us to immediately conclude that (5.10) holds true.

Step 2. We show that for any $u \in \mathcal{X}_{x}$ there exists a sequence $\left\{u_{n}\right\}_{n \in \mathbb{N}} \subset \mathcal{X}_{x}$ such that

$$
\lim _{n \rightarrow \infty} \sup _{t \leq 0}\left|u_{n}(t)-u(t)\right|_{H}=0
$$

and

$$
S(u) \geq \limsup _{n \rightarrow \infty} \tilde{S}\left(u_{n}\right) .
$$

If $S(u)=+\infty$, then (5.11) is trivially satisfied. Thus, let us assume that $S(u)=$ $r$. Our aim is to construct a sequence converging to $u$ in $\mathcal{X}_{x}$, such that (5.11) holds. First of all, notice that, as $S(u)=r$, we have

$$
\frac{\partial u}{\partial t}(t, \xi)=\mathcal{A} u(t, \xi)+b(\xi, u(t, \xi))+g(\xi, u(t, \xi)) \mathcal{H}(u)(t, \xi), \quad t \leq 0, \quad \xi \in \mathcal{O},
$$

with

$$
|\mathcal{H}(u)|_{L^{2}((-\infty, 0) ; H)}^{2}=2 r .
$$

Then, due to (5.7) $u \in L^{\infty}\left((-\infty, 0) ; D\left((-A)^{\frac{1}{2}}\right)\right)$ and estimate (5.8) holds true.

For any $\delta>0$, we consider the problem

$$
\left\{\begin{array}{l}
\frac{\partial v}{\partial t}(t, \xi)=\mathcal{A} v(t, \xi)+b(\xi, v(t, \xi))+Q_{\delta}[g(\cdot, u(t, \cdot)) \mathcal{H}(u)](t, \xi), \quad t>-\frac{1}{\delta}, \quad \xi \in \mathcal{O}, \\
v(-1 / \delta, \xi)=0, \quad \xi \in \mathcal{O}, \quad \mathcal{N} v(t, \xi)=0, \quad t>-\frac{1}{\delta}, \quad \xi \in \partial \mathcal{O}
\end{array}\right.
$$

and we denote by $v_{\delta}$ its unique mild solution in $C([-1 / \delta, 0] ; H)$. By using arguments similar (and simpler) to those used in [5] for the proof of Lemma 4.4 and for the proof of (4.19), it is possible to check that

$$
\sup _{t \in[-1 / \delta, 0]}\left|v_{\delta}(t)\right|_{\left.D\left((-A)^{\beta+\frac{1}{2}}\right)\right)} \leq c(1+\sqrt{2 r}),
$$


for some constant $c$ independent of $\delta>0$. In particular, as $\beta+1 / 2>d / 2$, due to the Sobolev embedding theorem

$$
\sup _{t \in[-1 / \delta, 0]}\left|v_{\delta}(t)\right|_{L^{\infty}(\mathcal{O})} \leq c(1+\sqrt{2 r}) .
$$

Next, for any $\delta, \rho>0$ we denote by $v_{\delta, \rho}$ the function

$$
v_{\delta, \rho}(t)=-\frac{t}{\rho} e^{(t+\rho) A} v_{\delta}(-\rho)+\frac{t+\rho}{\rho} e^{-t A} x, \quad t \in[-\rho, 0] .
$$

Clearly, $v_{\delta, \rho} \in C([-\rho, 0] ; H)$, with $v_{\delta, \rho}(-\rho)=v_{\delta}(-\rho)$ and $v_{\delta, \rho}(0)=x$. Moreover, thanks to (5.13) we have

$$
\sup _{t \in[-\rho, 0]}\left|v_{\delta, \rho}(t)\right|_{H} \leq c
$$

for some $c$ independent of $\delta, \rho>0$. Therefore, if we set for any $\rho, \delta>0$

$$
u_{\delta, \rho}(t)= \begin{cases}0, & t \leq-\frac{1}{\delta}, \\ v_{\delta}(t), & -\frac{1}{\delta} \leq t \leq-\rho, \\ v_{\delta, \rho}(t), & -\rho \leq t \leq 0,\end{cases}
$$

we have that $u_{\delta, \rho} \in C((-\infty, 0] ; H)$, with $u_{\delta, \rho}(t) \equiv 0$, for $t \leq-1 / \delta$ and $u_{\delta, \rho}(0)=x$, so that $\left\{u_{\delta, \rho}\right\}_{\delta, \rho>0} \subset \mathcal{X}_{x}$. Now, the proof of Step 2 follows if we show that $u_{\delta, \rho}$ converges to $u$ in $\mathcal{X}_{x}$, as $\delta, \rho$ go to zero, and (5.11) holds. These facts are proved in the following three lemmas.

Lemma 5.4. Assume that $x \in D\left((-A)^{\alpha}\right)$, with $\alpha>0$. We have

$$
\lim _{\delta, \rho \rightarrow 0} u_{\delta, \rho}=u \quad \text { in } \mathcal{X}_{x} .
$$

Proof. For any $t \leq-1 / \delta$ we have

$$
\left|u(t)-u_{\delta, \rho}(t)\right|_{H}=|u(t)|_{H} .
$$

Then, as $u \in \mathcal{X}_{x}$, we have that $|u(t)|_{H} \rightarrow 0$ as $t \rightarrow-\infty$, and hence for any $\epsilon>0$ we can fix $\delta_{1, \epsilon}>0$ such that

$$
\sup _{\rho>0} \sup _{t \in(-\infty,-1 / \delta]}\left|u(t)-u_{\delta, \rho}(t)\right|_{H}<\epsilon, \quad \delta \leq \delta_{1, \epsilon} .
$$

In the time interval $[-1 / \delta, 0]$, the function $h_{\delta}(t):=v_{\delta}(t)-u(t)$ solves the problem

$$
\left\{\begin{array}{l}
\frac{\partial h}{\partial t}(t, \xi)=\mathcal{A} h(t, \xi)+\left[b\left(\xi, v_{\delta}(t, \xi)\right)-b(\xi, u(t, \xi))\right]+\left(Q_{\delta}-I\right)[g(\cdot, u(t, \cdot)) \mathcal{H}(u)](t, \xi), \\
h(-1 / \delta, \xi)=-u(-1 / \delta, \xi), \quad \xi \in \mathcal{O}, \quad \mathcal{N} h(t, \xi)=0, \quad t>-\frac{1}{\delta}, \quad \xi \in \partial \mathcal{O} .
\end{array}\right.
$$

Then, if we set

$$
\gamma_{\delta}(t):=\int_{-\frac{1}{\delta}}^{t} e^{(t-s) A}\left(Q_{\delta}-I\right)[G(u(s)) \mathcal{H}(u)(s)] d s
$$

and $z_{\delta}(t):=h_{\delta}(t)-\gamma_{\delta}(t)$, with the usual arguments, we get

$$
\frac{1}{2}\left|z_{\delta}(t)\right|_{H}^{2} \leq-\frac{\lambda-L_{b}}{2}\left|z_{\delta}(t)\right|_{H}^{2}+c\left|\gamma_{\delta}(t)\right|_{H}^{2}
$$


By comparison, this yields

$$
\left|z_{\delta}(t)\right|_{H}^{2} \leq e^{-\left(\lambda-L_{b}\right)\left(t+\frac{1}{\delta}\right)}|u(-1 / \delta)|_{H}^{2}+c \int_{-\frac{1}{\delta}}^{t} e^{-(t-s)\left(\lambda-L_{b}\right)}\left|\gamma_{\delta}(s)\right|_{H}^{2} d s,
$$

so that it is immediate to get

$$
\left|u(t)-v_{\delta}(t)\right|_{H}^{2}=\left|h_{\delta}(t)\right|_{H}^{2} \leq c \sup _{t \leq 0}\left|\gamma_{\delta}(t)\right|_{H}^{2}+c|u(-1 / \delta)|_{H}^{2}, \quad t \in\left[-\frac{1}{\delta}, 0\right] .
$$

We have

$$
\sup _{t \leq 0}\left|\gamma_{\delta}(t)\right|_{H}^{2} \leq c\left|\left(Q_{\delta}-I\right)[G(u(s)) \mathcal{H}(u)(s)]\right|_{L^{2}((-\infty, 0) ; H)}^{2} .
$$

Then, as

$$
|\mathcal{H}(u)|_{L^{2}((-\infty, 0) ; H)}^{2}=2 r<\infty
$$

and, for any $s \leq 0$,

$$
\left|\left(Q_{\delta}-I\right)[G(u(s)) \mathcal{H}(u)(s)]\right|_{H} \leq c|\mathcal{H}(u)(s)|_{H}
$$

and

$$
\lim _{\delta \rightarrow 0}\left|\left(Q_{\delta}-I\right)[G(u(s)) \mathcal{H}(u)(s)]\right|_{H}=0,
$$

from the dominated convergence theorem we obtain

$$
\lim _{\delta \rightarrow 0} \sup _{t \leq 0}\left|\gamma_{\delta}(t)\right|_{H}^{2}=0 .
$$

Then, thanks to (5.18), we conclude that

$$
\lim _{\delta \rightarrow 0} \sup _{t \in[-1 / \delta, 0]}\left|u(t)-v_{\delta}(t)\right|_{H}=0 .
$$

In particular, as $u_{\delta, \rho}(t)=v_{\delta}(t)$ for $t \in\left[-\frac{1}{\delta},-\rho\right]$, this implies that there exists some $\delta_{2, \epsilon}>0$ such that

$$
\sup _{\rho>0} \sup _{t \in[-1 / \delta,-\rho]}\left|u(t)-u_{\delta, \rho}(t)\right|_{H}<\epsilon, \quad \delta \leq \delta_{2, \epsilon} .
$$

Now, we estimate $u_{\delta, \rho}(t)-u(t)$ for $t \in[-\rho, 0]$. We have

$$
\begin{aligned}
& u_{\delta, \rho}(t)-u(t)=-\frac{t}{\rho} e^{(t+\rho) A} v_{\delta}(-\rho)+\frac{t+\rho}{\rho} e^{-t A} x-u(t) \\
& =\frac{t}{\rho}\left(e^{-t A}-I\right) x+\frac{t}{\rho}\left(x-v_{\delta}(-\rho)\right)+\frac{t}{\rho}\left(I-e^{(t+\rho) A}\right) v_{\delta}(-\rho) \\
& +e^{-t A}(x-u(t))+\left(e^{-t A}-I\right) u(t)=: \sum_{i=1}^{5} I_{i}(t) .
\end{aligned}
$$

As we are assuming $x \in D\left((-A)^{\alpha}\right)$, for $\alpha>0$, and $t \in[-\rho, 0]$, we have

$$
\left|I_{1}(t)\right|_{H} \leq c|t|^{\alpha}|x|_{D\left((-A)^{\alpha}\right)} \leq \rho^{\alpha}|x|_{D\left((-A)^{\alpha}\right)}, \quad t \geq-\rho .
$$

In view of (5.20), as $v_{\delta}(-\rho)=u_{\delta, \rho}(-\rho)$, for any $\delta \leq \delta_{2, \epsilon}$

$$
\left|I_{2}(t)\right|_{H} \leq\left|x-v_{\delta}(-\rho)\right|_{H} \leq|x-u(-\rho)|_{H}+\epsilon, \quad t \geq-\rho .
$$

Concerning $I_{3}(t)$, according to (5.13) we have

$$
\left|I_{3}(t)\right|_{H} \leq c|t+\rho|^{\beta+\frac{1}{2}}\left|v_{\delta}(-\rho)\right|_{D\left((-A)^{\left.\beta+\frac{1}{2}\right)}\right.} \leq c|t+\rho|^{\beta+\frac{1}{2}} \leq c \rho^{\beta+\frac{1}{2}}, \quad t \geq-\rho .
$$


Next, we have

$$
\left|I_{4}(t)\right|_{H} \leq c \sup _{t \in[-\rho, 0]}|x-u(t)|_{H}, \quad t>-\rho,
$$

and finally, according to (5.8), we have

$$
\left|I_{5}(t)\right|_{H} \leq c|t|^{\frac{1}{2}}|u(t)|_{D\left((-A)^{\frac{1}{2}}\right)} \leq c \rho^{\frac{1}{2}}, \quad t>-\rho .
$$

Collecting together estimates from (5.21) to (5.25), for any $\delta \leq \delta_{2, \epsilon}$ and $\rho \leq 1$ we obtain

$$
\sup _{t \in[-\rho, 0]}\left|u_{\delta, \rho}(t)-u(t)\right|_{H} \leq c\left(\rho^{\alpha}|x|_{D\left((-A)^{\alpha}\right)}+\rho^{\frac{1}{2}}+\sup _{t \in[-\rho, 0]}|x-u(t)|_{H}+\epsilon\right),
$$

and this, together with (5.17) and (5.20), implies

$$
\limsup _{\rho \rightarrow 0} \sup _{t \leq 0}\left|u_{\delta, \rho}(t)-u(t)\right|_{H} \leq 3 \epsilon, \quad \delta \leq \delta_{\epsilon}:=\delta_{1, \epsilon} \wedge \delta_{2, \epsilon} .
$$

This yields (5.16), due to the arbitrariness of $\epsilon$.

Lemma 5.5. Assume that $x \in D\left((-A)^{\alpha}\right)$, with $\alpha>1 / 2$. Then there exist two sequences $\left\{\delta_{n}\right\}_{n \in \mathbb{N}}$ and $\left\{\rho_{n}\right\}_{n \in \mathbb{N}}$ both converging to zero, such that

$$
\lim _{n \rightarrow \infty} S\left(u_{\delta_{n}, \rho_{n}}\right)=S(u) .
$$

Proof. First of all, we compute $\mathcal{H}\left(v_{\delta, \rho}\right)(t)$ for $t \in[-\rho, 0]$. It is immediate to check that

$$
\frac{\partial v_{\delta, \rho}}{\partial t}-\mathcal{A} v_{\delta, \rho}=\frac{1}{\rho}\left[e^{-t A} x-e^{(t+\rho) A} v_{\delta}(-\rho)\right]-\frac{2(t+\rho)}{\rho} A e^{-t A} x,
$$

so that

$$
\begin{aligned}
\mathcal{H}\left(v_{\delta, \rho}\right)(t)=G^{-1}\left(v_{\delta, \rho}(t)\right)\left[\frac { 1 } { \rho } \left(e^{-t A} x-\right.\right. & \left.e^{(t+\rho) A} v_{\delta}(-\rho)\right) \\
& \left.-\frac{2(t+\rho)}{\rho} A e^{-t A} x-B\left(v_{\delta, \rho}(t)\right)\right] .
\end{aligned}
$$

We have

$$
\begin{aligned}
& \left|\frac{1}{\rho}\left(e^{-t A} x-e^{(t+\rho) A} v_{\delta}(-\rho)\right)-\frac{2(t+\rho)}{\rho} A e^{-t A} x\right|_{H}^{2} \leq \frac{4}{\rho^{2}}\left|e^{-t A}\left(x-v_{\delta}(0)\right)\right|_{H}^{2} \\
& +\frac{4}{\rho^{2}}\left|e^{-t A}\left(v_{\delta}(0)-v_{\delta}(-\rho)\right)\right|_{H}^{2}+\frac{4}{\rho^{2}}\left|\left(e^{-t A}-e^{(t+\rho) A}\right) v_{\delta}(-\rho)\right|_{H}^{2} \\
& +16\left|\frac{t+\rho}{\rho} A e^{-t A} x\right|_{H}^{2}=: \frac{4}{\rho^{2}}\left|e^{-t A}\left(x-v_{\delta}(0)\right)\right|_{H}^{2}+\sum_{i=1}^{3} I_{i}(\delta, \rho)(t) .
\end{aligned}
$$

Due to the properties of the analytic semigroup $e^{t A}$, it is possible to prove that for any $\theta \in(0,1)$ and $p>1 /(1-\theta)$ and for any $f \in L^{p}\left(\left(T_{1}, T_{2}\right) ; H\right)$ and $-\infty \leq T_{1} \leq$ $t \leq t+h \leq T_{2} \leq+\infty$

$$
\left|\int_{T_{1}}^{t+h} e^{(t+h-s) A} f(s) d s-\int_{T_{1}}^{t} e^{(t-s) A} f(s) d s\right|_{H} \leq c_{\theta, p} h^{\theta}|f|_{L^{p}\left(\left(T_{1}, T_{2}\right) ; H\right)}
$$


for some constant $c_{\theta, p}$ independent of $T_{1}$ and $T_{2}$. Now, due to (5.8) and (5.13), we have that $B\left(v_{\delta}(\cdot)\right)$ and $Q_{\delta}[G(u)(\cdot) \mathcal{H}(u)(\cdot)]$ are in $L^{\infty}((-1 / \delta, 0) ; H)$. Then, for any $\theta \in(0,1)$ we obtain

$$
\begin{array}{r}
\left|v_{\delta}(0)-v_{\delta}(-\rho)\right|_{H} \leq\left|\int_{-\frac{1}{\delta}}^{0} e^{-s A} B\left(v_{\delta}(s)\right) d s-\int_{-\frac{1}{\delta}}^{-\rho} e^{-(\rho+s) A} B\left(v_{\delta}(s)\right) d s\right|_{H} \\
+\left|\int_{-\frac{1}{\delta}}^{0} e^{-s A} Q_{\delta}[G(u(s)) \mathcal{H}(u)(s)] d s-\int_{-\frac{1}{\delta}}^{-\rho} e^{-(\rho+s) A} Q_{\delta}[G(u(s)) \mathcal{H}(u)(s)] d s\right|_{H} \\
\leq C_{\theta} \rho^{\theta},
\end{array}
$$

for a constant $c_{\theta}$ which is independent of $\delta>0$. This implies that for any $\theta \in$ $(1 / 2,1)$

$$
\sup _{\delta>0} I_{1}(\delta, \rho)(t) \leq c_{\theta} \rho^{2 \theta-2}, \quad t \in[-\rho, 0] .
$$

Concerning $I_{2}(\delta, \rho)(t)$, according to (5.13) we have

$$
\sup _{\delta>0} I_{2}(\delta, \rho)(t) \leq c \rho^{2 \beta-1}, \quad t \in[-\rho, 0],
$$

and concerning $I_{3}(\delta, \rho)(t)$, as $x \in D\left((-A)^{\alpha}\right)$, we obtain

$$
\sup _{\delta>0} I_{3}(\delta, \rho)(t) \leq c \rho^{2(\alpha-1)}, \quad t \in[-\rho, 0] .
$$

Therefore, thanks to (5.28), (5.29) and (5.30), we conclude that for any $\delta, \rho>0$

$$
\begin{aligned}
& \int_{-\rho}^{0}\left|G^{-1}\left(v_{\delta, \rho}(t)\right)\left[\frac{1}{\rho}\left(e^{-t A} x-e^{(t+\rho) A} v_{\delta}(-\rho)\right)-\frac{2(t+\rho)}{\rho} A e^{-t A} x\right]\right|_{H}^{2} d t \\
& \leq c\left(\rho^{2 \theta-1}+\rho^{2 \beta}+\rho^{2 \alpha-1}\right)+\frac{4}{\rho}\left|x-v_{\delta}(0)\right|_{H}^{2} .
\end{aligned}
$$

In view of (5.15) and (5.27), for any $\delta>0$ and $\rho \in(0,1)$ this yields

$$
\begin{aligned}
& \int_{-\rho}^{0}\left|\mathcal{H}\left(v_{\delta, \rho}\right)(t)\right|_{H}^{2} d t \leq c\left(\rho^{2 \theta-1}+\rho^{2 \beta}+\rho^{2 \alpha-1}\right)+\frac{c}{\rho}\left|x-v_{\delta}(0)\right|_{H}^{2} \\
& +c \int_{-\rho}^{0}\left|G^{-1}\left(v_{\delta, \rho}(t)\right) B\left(v_{\delta, \rho}(t)\right)\right|_{H}^{2} d t \leq c \rho^{\bar{\gamma}}+\frac{c}{\rho}\left|x-v_{\delta}(0)\right|_{H}^{2},
\end{aligned}
$$

where

$$
\bar{\gamma}:=\min \{2 \theta-1,2 \beta, 2 \alpha-1,1\}>0
$$

(notice that here $d \geq 2$, and hence $\beta>0$ ).

Now, since for any $\delta, \rho>0$

$$
S\left(u_{\delta, \rho}\right)=\frac{1}{2} \int_{-\frac{1}{\delta}}^{-\rho}\left|\frac{Q_{\delta}[G(u(s)) \mathcal{H}(u)(s)]}{g\left(\cdot, v_{\delta}(s, \cdot)\right)}\right|_{H}^{2} d s+\frac{1}{2} \int_{-\rho}^{0}\left|\mathcal{H}\left(v_{\delta, \rho}\right)(t)\right|_{H}^{2} d t,
$$


we get

$$
\begin{aligned}
& \left|S\left(u_{\delta, \rho}\right)-S(u)\right| \leq \frac{1}{2}\left|\int_{-\frac{1}{\delta}}^{-\rho}\left(\left|\frac{Q_{\delta}[G(u(s)) \mathcal{H}(u)(s)]}{g\left(\cdot, v_{\delta}(s, \cdot)\right)}\right|_{H}^{2}-|\mathcal{H}(u)(s)|_{H}^{2}\right) d s\right| \\
& +\frac{1}{2} \int_{-\rho}^{0}\left|\mathcal{H}\left(v_{\delta, \rho}\right)(t)\right|_{H}^{2} d t+\frac{1}{2} \int_{-\infty}^{-\frac{1}{\delta}}|\mathcal{H}(u)(s)|_{H}^{2} d s+\frac{1}{2} \int_{-\rho}^{0}|\mathcal{H}(u)(s)|_{H}^{2} d s .
\end{aligned}
$$

Due to (5.19) and (5.31), for any $\rho \in(0,1)$ there exists $\delta(\rho)>0$ such that

$$
\int_{-\rho}^{0}\left|\mathcal{H}\left(v_{\delta(\rho), \rho}\right)(t)\right|_{H}^{2} d t \leq c \rho^{\bar{\gamma}}+\frac{c}{\rho}\left|x-v_{\delta(\rho)}(0)\right|_{H}^{2} \leq c \rho^{\bar{\gamma}}
$$

and

$$
\lim _{\rho \rightarrow 0} \delta(\rho)=0
$$

Hence, as $\mathcal{H}(u) \in L^{2}((-\infty, 0) ; H)$, we obtain

$$
\lim _{\rho \rightarrow 0} \int_{-\rho}^{0}\left|\mathcal{H}\left(v_{\delta(\rho), \rho}\right)(t)\right|_{H}^{2} d t+\int_{-\infty}^{-\frac{1}{\delta(\rho)}}|\mathcal{H}(u)(s)|_{H}^{2} d s+\int_{-\rho}^{0}|\mathcal{H}(u)(s)|_{H}^{2} d s=0 .
$$

This means that, in order to conclude the proof of (5.26), we have to show that

$$
\lim _{\rho \rightarrow 0} \int_{-\frac{1}{\delta(\rho)}}^{-\rho}\left(\left|\frac{Q_{\delta(\rho)}[G(u(s)) \mathcal{H}(u)(s)]}{g\left(\cdot, v_{\delta(\rho)}(s, \cdot)\right)}\right|_{H}^{2}-|\mathcal{H}(u)(s)|_{H}^{2}\right) d s=0 .
$$

We have

$$
\begin{aligned}
& \left.|| \frac{Q_{\delta(\rho)}[G(u(s)) \mathcal{H}(u)(s)]}{g\left(\cdot, v_{\delta(\rho)}(s, \cdot)\right)}\right|_{H} ^{2}-|\mathcal{H}(u)(s)|_{H}^{2} \mid \\
& \leq c\left|\left(Q_{\delta(\rho)}-I\right)[G(u(s)) \mathcal{H}(u)(s)]\right|_{H}+c\left|\left[G\left(v_{\delta(\rho)}(s)\right)-G(u(s))\right] \mathcal{H}(u)(s)\right|_{H} .
\end{aligned}
$$

For any $s \leq 0$ we have

$$
\lim _{\rho \rightarrow 0} \mathbb{I}_{[-1 / \delta(\rho),-\rho]}(s)\left|\left(Q_{\delta(\rho)}-I\right)[G(u(s)) \mathcal{H}(u)(s)]\right|_{H}=0
$$

and then, as

$$
\mathbb{I}_{[-1 / \delta(\rho),-\rho]}(s)\left|\left(Q_{\delta(\rho)}-I\right)[G(u(s)) \mathcal{H}(u)(s)]\right|_{H} \leq c|\mathcal{H}(u)(s)|_{H}, \quad s \leq 0,
$$

by the dominated convergence theorem we conclude that

$$
\lim _{\rho \rightarrow 0} \int_{-\frac{1}{\delta(\rho)}}^{-\rho}\left|\left(Q_{\delta(\rho)}-I\right)[G(u(s)) \mathcal{H}(u)(s)]\right|_{H} d s=0
$$

Moreover, thanks to (5.19), it is possible to show that

$$
\lim _{\rho \rightarrow 0} \mathbb{I}_{[-1 / \delta(\rho),-\rho]}(s)\left|\left[G\left(v_{\delta(\rho)}(s)\right)-G(u(s))\right] \mathcal{H}(u)(s)\right|_{H}=0, \quad s \leq 0 .
$$

Then, as

$$
\mathbb{I}_{[-1 / \delta(\rho),-\rho]}(s)\left|\left[G\left(v_{\delta(\rho)}(s)\right)-G(u(s))\right] \mathcal{H}(u)(s)\right|_{H} \leq c|\mathcal{H}(u)(s)|_{H},
$$

again by the dominated convergence theorem we have

$$
\lim _{\rho \rightarrow 0} \int_{-\frac{1}{\delta(\rho)}}^{-\rho}\left|\left[G\left(v_{\delta(\rho)}(s)\right)-G(u(s))\right] \mathcal{H}(u)(s)\right|_{H} d s=0 .
$$

Together with (5.33), this implies (5.32) and (5.26) is proved. 
Lemma 5.6. Assume that $x \in D\left((-A)^{\alpha}\right)$, with $\alpha \geq \beta+1 / 2$. Then, for any $\delta, \rho>0$ it holds that

$$
S\left(u_{\delta, \rho}\right)=\tilde{S}\left(u_{\delta, \rho}\right) .
$$

Proof. In order to prove (5.34), we have to show that $u_{\delta, \rho} \in \mathcal{Y}_{x}$, for any $\delta, \rho>0$. Due to the definition of $u_{\delta, \rho}$, we have that

$$
\frac{\partial u_{\delta, \rho}}{\partial t}(t, \xi)=\mathcal{A} u_{\delta, \rho}(t, \xi)+b\left(\xi, u_{\delta, \rho}(t, \xi)\right)+g\left(\xi, u_{\delta, \rho}(t, \xi)\right) K_{\delta, \rho}(t, \xi),
$$

where

$$
K_{\delta, \rho}(t)= \begin{cases}0 & t<-1 / \delta \\ G^{-1}\left(v_{\delta}(t)\right)\left(Q_{\delta}[G(u(t)) \mathcal{H}(u)(t)]\right), & -1 / \delta \leq t \leq-\rho, \\ \mathcal{H}\left(v_{\delta, \rho}\right)(t), & -\rho<t \leq 0\end{cases}
$$

We need to show that

$$
\int_{-\infty}^{0}\left|Q_{1}^{-1} K_{\delta, \rho}(t)\right|_{H}^{2} d t<\infty
$$

Due to (2.15) and (2.16), we have

$$
\left|Q_{\delta}[G(u(t)) \mathcal{H}(u)(t)]\right|_{D\left((-A)^{\beta}\right)} \leq c_{\delta}|G(u(t)) \mathcal{H}(u)(t)|_{H} \leq c_{\delta}|\mathcal{H}(u)(t)|_{H}, \quad t \leq 0 .
$$

Therefore, thanks to (2.4), we have that $Q_{\delta}[G(u(\cdot)) \mathcal{H}(u)] \in L^{2}\left((-\infty, 0) ; H_{\mathcal{N}_{\beta}}^{2 \beta, 2}(\mathcal{O})\right)$ and

$$
\left|Q_{\delta}[G(u(\cdot)) \mathcal{H}(u)]\right|_{L^{2}\left((-\infty, 0) ; H_{\mathcal{N}_{\beta}}^{2 \beta, 2}(\mathcal{O})\right)} \leq c_{\delta}
$$

Moreover, as $1 / g \in C^{\infty}(\bar{D} \times \mathbb{R})$ and, according to (5.13),

$$
\begin{aligned}
v_{\delta} \in L^{\infty}\left((-1 / \delta, 0) ; D\left((-A)^{\beta+\frac{1}{2}}\right)\right) & =L^{\infty}\left((-1 / \delta, 0) ; H_{\left.\mathcal{N}_{\beta+\frac{1}{2}}^{2 \beta+1,2}(\mathcal{O})\right)}\right. \\
& \subset L^{\infty}\left((-1 / \delta, 0) ; L^{\infty}(\mathcal{O})\right),
\end{aligned}
$$

from [12, Theorem 5.5.4.1] and (2.29) we obtain that

$$
\frac{1}{g\left(\cdot, v_{\delta}\right)} \in L^{\infty}\left((-1 / \delta, 0) ; H_{\mathcal{N}_{\beta+\frac{1}{2}}^{2 \beta+1,2}}(\mathcal{O})\right) \text {. }
$$

Since $2 \beta+1>d / 2$, in view of (2.1) and (5.36) this implies

$$
G^{-1}\left(v_{\delta}(t)\right)\left(Q_{\delta}[G(u(t)) \mathcal{H}(u)(t)]\right) \in H^{2 \beta, 2}(\mathcal{O}) .
$$

Moreover, thanks to (2.29), we have that

$$
\mathcal{N}_{\beta}\left[G^{-1}\left(v_{\delta}(t)\right)\left(Q_{\delta}[G(u(t)) \mathcal{H}(u)(t)]\right)\right]_{\left.\right|_{\text {○O }}}=0,
$$

so that $G^{-1}\left(v_{\delta}(t)\right)\left(Q_{\delta}[G(u(t)) \mathcal{H}(u)(t)]\right) \in H_{\mathcal{N}_{\beta}}^{2 \beta, 2}(\mathcal{O})=D\left((-A)^{\beta}\right)$ and, thanks to (2.17) and (5.36),

$$
\begin{aligned}
& \left|Q_{1}^{-1}\left(G^{-1}\left(v_{\delta}\right)\left(Q_{\delta}[G(u) \mathcal{H}(u)]\right)\right)\right|_{L^{2}((-1 / \delta ; 0) ; H)} \\
& \leq c_{1}\left|G^{-1}\left(v_{\delta}\right)\left(Q_{\delta}[G(u) \mathcal{H}(u)]\right)\right|_{L^{2}\left((-1 / \delta ; 0) ; D\left((-A)^{\beta}\right)\right)} \leq c_{\delta} .
\end{aligned}
$$

Concerning $\mathcal{H}\left(v_{\delta, \rho}\right)$, in (5.27) we have seen that

$$
\mathcal{H}\left(v_{\delta, \rho}\right)(t)=G^{-1}\left(v_{\delta, \rho}(t)\right) \psi_{\delta, \rho}(t),
$$


where

$$
\psi_{\delta, \rho}(t):=\frac{1}{\rho}\left(e^{-t A} x-e^{(t+\rho) A} v_{\delta}(-\rho)\right)-\frac{2(t+\rho)}{\rho} A e^{-t A} x-B\left(v_{\delta, \rho}(t)\right) .
$$

Then, by proceeding as above, in order to prove that

$$
\left|Q_{1}^{-1} \mathcal{H}\left(v_{\delta, \rho}\right)\right|_{L^{2}((-\rho, 0) ; H)}<\infty
$$

and hence conclude the proof of (5.35), we have to show that

$$
\psi_{\delta, \rho} \in L^{2}\left((-\rho, 0) ; D\left((-A)^{\beta}\right)\right)=L^{2}\left((-\rho, 0) ; H_{\mathcal{N}_{\beta}}^{2 \beta, 2}(\mathcal{O})\right) .
$$

If $x \in D\left((-A)^{\alpha}\right)$ with $\alpha \geq \beta+1 / 2$, it is immediate to check that

$$
\int_{-\rho}^{0}\left(\left|(-A)^{\beta} e^{-t A} x\right|_{H}^{2}+\left|(-A)^{\beta+1} e^{-t A} x\right|_{H}^{2}\right) d t \leq c_{\rho, \alpha}|x|_{D\left((-A)^{\alpha}\right)}^{2} .
$$

Moreover, according to (5.13), we have

$$
\int_{-\rho}^{0}\left|(-A)^{\beta} e^{-(t+\rho) A} v_{\delta}(-\rho)\right|_{H}^{2} d t \leq c_{\rho}
$$

Finally, since

$$
v_{\delta, \rho} \in L^{\infty}\left((-\rho, 0) ; D\left((-A)^{\beta+\frac{1}{2}}\right)\right) \subset L^{\infty}\left((-\rho, 0) ; L^{\infty}(\mathcal{O})\right)
$$

and $b \in C^{\infty}(\overline{\mathcal{O}} \times \mathbb{R})$, by proceeding as before for $1 / g$, due to [12, Theorem 5.5.4.1] we have that $B\left(v_{\delta, \rho}\right)(t) \in D\left((-A)^{\beta+\frac{1}{2}}\right)$ and

$$
\left|B\left(v_{\delta, \rho}\right)(t)\right|_{D\left((-A)^{\beta+\frac{1}{2}}\right)} \leq c\left(1+\left|v_{\delta, \rho}(t)\right|_{\infty}^{k}\right)\left|v_{\delta, \rho}\right|_{D\left((-A)^{\beta+\frac{1}{2}}\right)}
$$

for some power $k \geq 0$. This implies that

$$
\int_{-\rho}^{0}\left|(-A)^{\beta} B\left(v_{\delta, \rho}(t)\right)\right|_{H}^{2} d t \leq c_{\rho},
$$

so that from (5.39) and (5.40) we can conclude that (5.38) holds true. This finishes the proof of the lemma.

\section{THE QUASI-POTENTIAL}

On the space $C\left(\left[T_{1}, T_{2}\right] ; H\right)$ with $-\infty \leq T_{1}<T_{2} \leq+\infty$, we introduce the operator

$$
S_{T_{1}, T_{2}}(u)= \begin{cases}\frac{1}{2} \int_{T_{1}}^{T_{2}}|\mathcal{H}(u)|_{H}^{2} d t, & \text { if } u \in W_{2, \mathcal{N}}^{1,2}\left(T_{1}, T_{2}\right), \\ +\infty, & \text { otherwise, }\end{cases}
$$

where $W_{2, \mathcal{N}}^{1,2}\left(T_{1}, T_{2}\right)$ is the subspace of $C\left(\left[T_{1}, T_{2}\right] ; H\right)$ introduced in (5.2) and $\mathcal{H}$ is the operator introduced in (5.1).

For any $x \in H$ we define

$$
V(x):=\inf \left\{S_{0, T}(u): u \in C([0, T] ; H), T>0, u(0)=0, u(T)=x\right\} .
$$


In Proposition 5.1 we have shown that the level sets of $S$ (and hence of $S_{-\infty, 0}$ ) are compact in $C((-\infty, 0) ; H)$. Thus, by proceeding as in the proof of 13 , Proposition $7]$, it is possible to show that $V$ can be characterized as

$$
V(x)=\min \left\{S_{-\infty, 0}(u): u \in C((-\infty, 0] ; H), \lim _{t \rightarrow-\infty}|u(t)|_{H}=0, u(0)=x\right\},
$$

for any $x \in H$. Recalling how the operator $S$ was defined in (5.5), this means that

$$
V(x)=\min \left\{S(u): u \in \mathcal{X}_{x}\right\}
$$

In [13, Sowers has considered equation (2.30) in space dimension $d=1$, with $Q_{\delta}=I$, and he has proved that the family of invariant measures $\left\{\nu_{\epsilon}\right\}_{\epsilon>0}$, which we have introduced at the end of Section 2, obeys a large deviation principle with the action functional given by the quasi-potential $V$.

Next, for any $\delta>0$, we introduce the operator

$$
S_{T_{1}, T_{2}}^{\delta}(u)= \begin{cases}\frac{1}{2} \int_{T_{1}}^{T_{2}}\left|Q_{\delta}^{-1} \mathcal{H}(u)\right|_{H}^{2} d t, & \text { if } u \in W_{2, \mathcal{N}}^{1,2}\left(T_{1}, T_{2}\right), \\ +\infty, & \text { otherwise }\end{cases}
$$

and for any $x \in H$ we define

$$
V_{\delta}(x):=\inf \left\{S_{0, T}^{\delta}(u): u \in C([0, T] ; H), T>0, u(0)=0, u(T)=x\right\} .
$$

In [5, Proposition 5.4] we have proved that $V_{\delta}$ can be characterized as

$$
V_{\delta}(x)=\min \left\{S_{-\infty, 0}^{\delta}(u): u \in C((-\infty, 0] ; H), \lim _{t \rightarrow-\infty}|u(t)|_{H}=0, u(0)=x\right\},
$$

for any $x \in H$, so that, recalling how the operator $S_{\delta}$ was defined in (5.3), we have

$$
V_{\delta}(x)=\min \left\{S_{\delta}(u): u \in \mathcal{X}_{x}\right\} .
$$

In particular, in view of Lemma 4.4 this implies

$$
V_{\delta}(x)<\infty \Longleftrightarrow x \in D\left((-A)^{\beta+\frac{1}{2}}\right) .
$$

In the general case considered by Sowers in [13], unlike $S_{T_{1}, T_{2}}$ which is explicitly given, the quasi-potential $V$ is given implicitly as an infimum. As shown in [7, there are some cases in which $V$ is explicitly given. The first one is the case of a linear equation perturbed by an additive noise

$$
\left\{\begin{array}{l}
\frac{\partial u}{\partial t}(t, \xi)=\mathcal{A} u(t, \xi)+\frac{\partial w^{Q}}{\partial t}(t, \xi), \\
u(0, \xi)=x(\xi), \quad \mathcal{N} u(t, \xi)=0, \quad \xi \in \partial \mathcal{O} .
\end{array}\right.
$$

In this case, under the assumption that the linear operator

$$
R:=\int_{0}^{\infty} e^{t A} Q Q^{\star} e^{t A^{\star}} d t
$$

is bounded and nuclear on $H$, we have

$$
V(x)=\frac{1}{2}\left|R^{-\frac{1}{2}} x\right|_{H}^{2}, \quad x \in H,
$$

with the usual convention that if an element $x$ is not in the domain of an operator $C$, then $|C x|_{H}=+\infty$ (for a proof see [7]). In the case where $A$ and $Q$ are diagonal 
with respect to the same orthonormal basis $\left\{e_{k}\right\}_{k}$ of $H$, with eigenvalues $\left\{\alpha_{k}\right\}_{k \in \mathbb{N}}$ and $\left\{\lambda_{k}\right\}_{k \in \mathbb{N}}$, respectively, the operator $R$ can be computed and we have

$$
R x=\frac{1}{2} \sum_{k=1}^{\infty} \frac{\lambda_{k}^{2}}{\alpha_{k}}\left\langle x, e_{k}\right\rangle_{H} e_{k},
$$

so that

$$
V(x)=\sum_{k=1}^{\infty} \frac{\alpha_{k}}{\lambda_{k}^{2}}\left|\left\langle x, e_{k}\right\rangle_{H}\right|^{2}
$$

In particular, we have

$$
Q=(-A)^{-\beta}, \beta \geq 0 \Longrightarrow V(x)=\left|(-A)^{\frac{1}{2}+\beta} x\right|_{H}^{2} .
$$

This means that if $V$ and $V_{\delta}$ are the functionals defined, respectively, in (6.2) and (6.5), we have

$$
V(x)= \begin{cases}\left|(-A)^{\frac{1}{2}} x\right|_{H} & \text { if } x \in D\left((-A)^{\frac{1}{2}}\right) \\ +\infty & \text { otherwise }\end{cases}
$$

and

$$
V_{\delta}(x)= \begin{cases}\sum_{k=1}^{\infty} \frac{\alpha_{k}}{\lambda_{\delta, k}^{2}}\left|\left\langle x, e_{k}\right\rangle_{H}\right|^{2} & \text { if } x \in D\left((-A)^{\beta+\frac{1}{2}}\right), \\ +\infty & \text { otherwise. }\end{cases}
$$

Therefore, due to (2.12), we get

$$
\lim _{\delta \rightarrow 0} V_{\delta}(x)=V(x), \quad x \in D\left((-A)^{\beta+\frac{1}{2}}\right) .
$$

There is another relevant situation in which $V$ can be explicitly computed, and this is the case in which (2.30) is a gradient system; that is, $\mathcal{O}=(0, L)$ and

$$
\left\{\begin{array}{l}
\frac{\partial u}{\partial t}(t, \xi)=\mathcal{A} u(t, \xi)+b(\xi, u(t, \xi))+\frac{\partial w}{\partial t}(t, \xi), \\
u(0, \xi)=x(\xi), \quad \mathcal{N} u(t, 0)=\mathcal{N} u(t, L)=0 .
\end{array}\right.
$$

In particular, $g(\xi, \sigma) \equiv 1$ and $Q_{\delta}=I$. In this case, as shown e.g. in 7, Theorem 3.7], it is possible to prove that $V$ is the potential associated with equation (2.30); that is,

$$
V(x)=\frac{1}{2}\left|(-A)^{\frac{1}{2}} x\right|_{H}^{2}-\int_{\mathcal{O}} \int_{0}^{x(\xi)} b(\xi, \sigma) d \sigma d \xi .
$$

We would like to stress that the functional $V(x)$ given in (6.12) coincides with the quasi-potential only in space dimension $d=1$, where we can take a white noise both in time and in space. Nevertheless, the expression of $V(x)$ given in (6.12) is also meaningful in space dimension $d \geq 1$, for any $x \in D\left((-A)^{\frac{1}{2}}\right)$. Moreover, as proved in [6, Theorem 12.23], for any $d \geq 1$

$$
\begin{aligned}
& \frac{1}{2}\left|(-A)^{\frac{1}{2}} x\right|_{H}^{2}-\int_{\mathcal{O}} \int_{0}^{x(\xi)} b(\xi, \sigma) d \sigma d \xi \\
& =\inf \left\{S_{0, T}(u), u \in C([0, T] ; H), u(0)=0, u(T)=x, T>0\right\},
\end{aligned}
$$


and hence, as we have already seen at the beginning of this section,

$$
\frac{1}{2}\left|(-A)^{\frac{1}{2}} x\right|_{H}^{2}-\int_{\mathcal{O}} \int_{0}^{x(\xi)} b(\xi, \sigma) d \sigma d \xi=\min \left\{S(u), u \in \mathcal{X}_{x}\right\} .
$$

In (6.10) we have seen that in the linear case $V_{\delta}(x)$ converges to $V(x)$, as $\delta$ goes to zero, for any $x \in D\left((-A)^{\beta+\frac{1}{2}}\right)$. In view of Theorem 5.3. we can prove that the same result is also true in the general case we have considered in the present paper.

Theorem 6.1. Assume Hypotheses 11 and 2, Then

$$
\lim _{\delta \rightarrow 0} V_{\delta}(x)=V(x), \quad x \in D\left((-A)^{\beta+\frac{1}{2}}\right) .
$$

Proof. In Theorem 5.3 we have shown that for any $x \in D\left((-A)^{\beta+\frac{1}{2}}\right)$

$$
\Gamma-\lim _{\delta \rightarrow 0} S_{\delta}=S \quad \text { in } \mathcal{X}_{x} .
$$

Then, according to Theorem 3.8, in order to get (6.13) we have to prove that the family $\left\{S_{\delta}\right\}_{\delta>0}$ is equi-coercive in $\mathcal{X}_{x}$. Actually, this implies that $S$ is coercive (but this we already knew) and, due to (6.3) and (6.6),

$$
V(x)=\min \left\{S(u): u \in X_{x}\right\}=\lim _{\delta \rightarrow 0} \inf _{u \in \mathcal{X}_{x}} S_{\delta}(u)=\lim _{\delta \rightarrow 0} V_{\delta}(x) .
$$

Thanks to Proposition 3.3 the equi-coerciveness of the family $\left\{S_{\delta}\right\}_{\delta>0}$ follows once we prove that there exists a lower semicontinuous and coercive function $\Psi: \mathcal{X}_{x} \rightarrow \overline{\mathbb{R}}$ such that

$$
S_{\delta}(u) \geq \Psi(u), \quad u \in \mathcal{X}_{x}, \quad \delta>0 .
$$

If $u \in \mathcal{X}_{x} \backslash \mathcal{Y}_{x}$, then $S_{\delta}(u)=+\infty$. If $u \in \mathcal{Y}_{x}$, due to (2.20) we have

$$
S_{\delta}(u)=\frac{1}{2} \int_{-\infty}^{0}\left|Q_{\delta}^{-1} \mathcal{H}(u)(s)\right|_{H}^{2} d s \geq \frac{1}{2} \int_{-\infty}^{0}|\mathcal{H}(u)(s)|_{H}^{2} d s=S(u) .
$$

Then, if we take $\Psi=S$, as $S$ is lower semicontinuous and coercive, we get (6.14) and then (6.13) follows.

\section{Application to the problem of the exit time FROM A BASIN OF ATTRACTION}

Let $D$ be a bounded subset of $H$. In what follows we denote by $u^{x}$ the solution of the unperturbed problem (4.1) starting from $x$, and we assume that if $x \in D$, then $u^{x}(t) \in D$, for any $t \geq 0$, and

$$
\lim _{t \rightarrow \infty}\left|u^{x}(t)\right|_{H}=0 .
$$

In view of (4.2), we have that the condition above is satisfied by any ball $B_{c, \gamma}=$ $\left\{x \in D\left((-A)^{\gamma}\right):|x|_{D\left((-A)^{\gamma}\right)} \leq c\right\}$, with $\gamma \leq 1 / 2$ and $c>0$, and also by any ball $B_{c, \infty}=\left\{x \in L^{\infty}(\mathcal{O}):|x|_{\infty} \leq c\right\}$, with $c>0$.

Moreover, as we are assuming $D$ to be bounded, there exists $c>0$ such that $\bar{D} \subseteq B_{c, 0}$. Thus, thanks to Lemma 4.2, we have that for any $\gamma \geq 0$ and $\mu>0$ there exists $T_{1}=T_{1}(\gamma, \mu)>0$ such that

$$
t_{\mu, \gamma}^{x}=\inf \left\{t \geq 0: u^{x}(t) \in B_{\mu, \gamma} \cap B_{\mu, \infty}\right\}<T_{1}, \quad x \in \bar{D},
$$

and

$$
u^{x}(t) \in B_{\mu, \gamma}, \quad t>T_{1} .
$$


Now, in correspondence with the domain $D$ and the initial point $x \in D$, for any $\epsilon, \delta>0$ we define the exit time

$$
\tau_{\epsilon, \delta}^{x}:=\inf \left\{t \geq 0: u_{\epsilon, \delta}^{x}(t) \in \partial D\right\},
$$

where $u_{\epsilon, \delta}^{x}$ is the solution of (2.30). In what follows we want to prove that, as in the finite dimensional case, the following limiting result holds.

Theorem 7.1. Assume that for any $\delta>0$, there exists $y_{\delta} \in \partial D$ such that

$$
V_{\delta}\left(y_{\delta}\right)=\inf _{x \in \partial D} V_{\delta}(x)=\min _{x \in \partial D} V_{\delta}(x) .
$$

Then, under Hypotheses 1 and 2, we have

$$
\lim _{\epsilon \rightarrow 0} \epsilon \log \mathbb{E} \tau_{\epsilon, \delta}^{x}=\inf _{y \in \partial D} V_{\delta}(y) .
$$

As in the finite dimensional case (see [10, proof of Theorem 4.1] and also [9, proof of Theorem 5.7.11]), the proof of the theorem above is a consequence of the following preliminary results, whose proofs are postponed until Appendix $\mathrm{A}$. Notice that in all these lemmas the positive parameter $\delta$ is fixed.

Lemma 7.2. For any $\eta>0$ and $\mu>0$, we can fix $\bar{T}>0$ and $h>0$ such that if $x \in B_{\mu, 0}$, then there exist $\bar{T}(x) \leq \bar{T}$ and $z^{x} \in C([0, \bar{T}(x)] ; H)$ with $z^{x}(0)=x$, such that

$$
d_{H}\left(z^{x}(\bar{T}(x)), \bar{D}\right)>h
$$

and

$$
S_{0, \bar{T}(x)}^{\delta}\left(z^{x}\right) \leq V_{\delta}\left(y_{\delta}\right)+\eta=\inf _{y \in \partial D} V(y)+\eta .
$$

Lemma 7.3. There exists $\bar{\mu}>0$ such that, for any $\eta>0$ and $0<\mu \leq \bar{\mu}$, there exists $\bar{T}>0$ such that

$$
\liminf _{\epsilon \rightarrow 0} \epsilon \log \inf _{x \in B_{\mu, \infty}} \mathbb{P}\left(\tau_{\epsilon, \delta}^{x} \leq \bar{T}\right)>-\left(\inf _{y \in \partial D} V_{\delta}(y)+\eta\right) .
$$

Lemma 7.4. For any $\epsilon>0$ and $\mu>0$ such that $B_{\mu, 0} \subseteq D$, let us define

$$
\sigma_{\epsilon, \delta, \mu}^{x}:=\inf \left\{t \geq 0 ; u_{\epsilon, \delta}^{x}(t) \in B_{\mu, 0} \cup \partial D\right\} .
$$

Then, for any $x \in D$

$$
\lim _{t \rightarrow \infty} \liminf _{\epsilon \rightarrow 0} \epsilon \log \mathbb{P}\left(\sigma_{\epsilon, \delta, \mu}^{x}>t\right)=-\infty .
$$

Lemma 7.5. For any $\mu>0$, with $B_{\mu, 0} \subseteq D$, and $x \in D$ we have

$$
\lim _{\epsilon \rightarrow 0} \mathbb{P}\left(u_{\epsilon, \delta}^{x}\left(\sigma_{\epsilon, \delta, \mu}^{x}\right) \in B_{\mu, 0}\right)=1 .
$$

Lemma 7.6. For every $\rho, \lambda>0$ and $x \in D$ there exists $\bar{T}=T(\rho, \lambda, x)<\infty$ such that

$$
\limsup _{\epsilon \rightarrow 0} \epsilon \log \mathbb{P}\left(\sup _{t \in[0, \bar{T}]}\left|u_{\epsilon, \delta}^{x}(t)-x\right|_{H} \geq \rho\right)<-\lambda .
$$

Now, in view of Theorem 6.1 we want to prove that the infimum of $V_{\delta}$ on $\partial D$ converges to the infimum of $V$ on $\partial D$, as the parameter $\delta$ goes to zero. 
Theorem 7.7. Assume that for any $x \in D\left((-A)^{\frac{1}{2}}\right) \cap \partial D$ there exists a sequence $\left\{x_{n}\right\}_{n \in \mathbb{N}} \subset D\left((-A)^{\beta+\frac{1}{2}}\right) \cap \partial D$ such that

$$
\lim _{n \rightarrow \infty}\left|x_{n}-x\right|_{D\left((-A)^{\frac{1}{2}}\right)}=0 .
$$

Then, under Hypotheses 1 and 2, we have

$$
\lim _{\delta \rightarrow 0} \inf _{x \in \partial D} V_{\delta}(x)=\inf _{x \in \partial D} V(x)
$$

In particular, we have

$$
\lim _{\delta \rightarrow 0} \lim _{\epsilon \rightarrow 0} \epsilon \log \mathbb{E} \tau_{\epsilon, \delta}^{x}=\inf _{x \in \partial D} V(x),
$$

so that, for small $\delta>0$,

$$
\mathbb{E} \tau_{\epsilon, \delta}^{x} \approx \exp \left(\frac{1}{\epsilon} \inf _{x \in \partial D} V(x)\right), \quad \text { as } \epsilon \downarrow 0 .
$$

Proof. For any $\delta>0$ we define

$$
\tilde{V}_{\delta}(x):= \begin{cases}V_{\delta}(x), & x \in D\left((-A)^{\beta+\frac{1}{2}}\right) \cap \partial D=: \partial D_{\beta}, \\ +\infty, & x \in H \backslash \partial D_{\beta}\end{cases}
$$

and, analogously, we define

$$
\tilde{V}(x):= \begin{cases}V(x), & x \in \partial D_{\beta}, \\ +\infty, & x \in H \backslash \partial D_{\beta} .\end{cases}
$$

According to Theorem 6.1 we have

$$
\lim _{\delta \rightarrow 0} \tilde{V}_{\delta}(x)=\tilde{V}(x), \quad x \in H .
$$

Moreover, as $S_{\delta_{1}}(u) \leq S_{\delta_{2}}(u)$, for any $\delta_{1}<\delta_{2}$ and $u \in \mathcal{X}_{x}$, we immediately have

$$
\delta_{1}<\delta_{2} \Longrightarrow \tilde{V}_{\delta_{1}}(x) \leq \tilde{V}_{\delta_{2}}(x), \quad x \in H
$$

Thus, thanks to Proposition 3.7, we have that

$$
\Gamma-\lim _{\delta \rightarrow 0} \tilde{V}_{\delta}=s c^{-} \tilde{V} .
$$

Since $S_{\delta}(u) \geq S(u)$, for any $\delta>0$ and $u \in \mathcal{X}_{x}$, we have

$$
\tilde{V}_{\delta}(x) \geq V_{\delta}(x)=\min \left\{S_{\delta}(u): u \in \mathcal{X}_{x}\right\} \geq \min \left\{S(u): u \in \mathcal{X}_{x}\right\}=V(x) .
$$

As $V(x)$ is lower semicontinuous and coercive, this implies the equi-coerciveness of the family $\left\{\tilde{V}_{\delta}\right\}_{\delta>0}$. Therefore, if we are able to prove that

$$
s c^{-} \tilde{V}(x)=\hat{V}(x):= \begin{cases}V(x), & x \in \partial D, \\ +\infty, & x \in H \backslash \partial D,\end{cases}
$$

as a consequence of Theorem 3.8 we have that (7.10) follows. In fact, due to (7.12) and (7.13), we have

$$
\lim _{\delta \rightarrow 0} \inf _{x \in H} \tilde{V}_{\delta}(x)=\inf _{x \in H} \hat{V}(x)=\inf _{x \in \partial D} V(x) .
$$

On the other side, as $V_{\delta}(x)=+\infty$, for any $x \in H \backslash D\left((-A)^{\beta+\frac{1}{2}}\right)$, we have

$$
\inf _{x \in H} \tilde{V}_{\delta}(x)=\inf _{x \in \partial D_{\beta}} V_{\delta}(x)=\inf _{x \in \partial D} V_{\delta}(x),
$$


and hence we can conclude that

$$
\lim _{\delta \rightarrow 0} \inf _{x \in \partial D} V_{\delta}(x)=\inf _{x \in \partial D} V(x) .
$$

Now, let us prove (7.13). We use the characterization of $s c^{-} \tilde{V}$ given in Proposition 3.5. Let $x \in H$ and let $\left\{x_{n}\right\} \subset H$ be any sequence converging to $x$. As $\partial D$ is closed in $H$, we have that $\hat{V}$ is lower semicontinuous. Actually, as proved in [5, Theorem 5.5], for any $r>0$ the set $\{V \leq r\}$ is compact in $H$ and in particular it is closed. Then, as

$$
\{\hat{V} \leq r\}=\{V \leq r\} \cap \partial D,
$$

we have that $\{\hat{V} \leq r\}$ is compact and in particular closed, so that $\hat{V}$ is lowersemicontinuous. This implies

$$
\hat{V}(x) \leq \liminf _{n \rightarrow \infty} \hat{V}\left(x_{n}\right) \leq \liminf _{n \rightarrow \infty} \tilde{V}\left(x_{n}\right) .
$$

Next, for any $x \in H$, we want to find a sequence $\left\{x_{n}\right\} \subset H$ converging to $x$ in $H$, such that

$$
\hat{V}(x) \geq \limsup _{n \rightarrow \infty} \tilde{V}\left(x_{n}\right) .
$$

If $\hat{V}(x)=+\infty$, there is nothing to prove. Then, assume $\hat{V}(x)<+\infty$. This means that $x \in D\left((-A)^{\frac{1}{2}}\right) \cap \partial D$. As we are assuming that there exists a sequence $\left\{x_{n}\right\} \subset D\left((-A)^{\beta+\frac{1}{2}}\right) \cap \partial D$ which converges to $x$ in the $D\left((-A)^{\frac{1}{2}}\right)$-norm, we conclude that

$$
\hat{V}(x)=V(x)=\lim _{n \rightarrow \infty} V\left(x_{n}\right)=\lim _{n \rightarrow \infty} \tilde{V}\left(x_{n}\right) .
$$

In view of (7.14) and (7.15), we can apply Proposition 3.5 and we get (7.13), which implies (7.10). Finally, (7.11) is a consequence of (7.10) and of Theorem 7.1 .

Remark 7.8. If $D=B_{c, 0}$, then $\partial D=\left\{x \in H ;|x|_{H}=c\right\}$. Thus, it is possible to prove that for any $x \in D\left((-A)^{\frac{1}{2}}\right) \cap \partial D$ there exists a sequence $\left\{x_{n}\right\}_{n \in \mathbb{N}} \subset$ $D\left((-A)^{\beta+\frac{1}{2}}\right) \cap \partial D$ converging to $x$ in the $D\left((-A)^{\frac{1}{2}}\right)$-norm. Actually, we fix a sequence $\left\{\hat{x}_{n}\right\} \subset D\left((-A)^{\beta+\frac{1}{2}}\right)$ such that

$$
\left|\hat{x}_{n}-x\right|_{D\left((-A)^{\frac{1}{2}}\right)}=0,
$$

and we define

$$
x_{n}:=c \frac{\hat{x}_{n}}{\left|\hat{x}_{n}\right|_{H}}, \quad n \in \mathbb{N} .
$$

Clearly $x_{n} \in \partial D$ and, as $\left|\hat{x}_{n}\right|_{H} \rightarrow|x|_{H}=c$, we have that

$$
\left|x_{n}-x\right|_{D\left((-A)^{\frac{1}{2}}\right)}=0 .
$$

\section{Appendix A}

Here we give a proof for all Lemmas from 7.2 to 7.6 . The proofs of Lemmas 7.3 , 7.4. 7.5 and 7.6 are very similar to the proofs of the corresponding finite dimensional results, once one has all the preliminary results already proved e.g. in 4 . On the contrary, the proof of Lemma 7.2 is much more delicate than in finite dimension and requires some extra work. 
A.1. Proof of Lemma 7.2. If $V_{\delta}\left(y_{\delta}\right)=+\infty$, there is nothing to prove. Thus, we can assume that $V_{\delta}\left(y_{\delta}\right)<\infty$, so that, in view of (6.7), we have that $y_{\delta} \in$ $D\left((-A)^{\beta+\frac{1}{2}}\right)$. Now, we fix $\hat{y}_{\delta} \in D\left((-A)^{\beta+\frac{1}{2}}\right)$ such that

$$
\left|\hat{y}_{\delta}-y_{\delta}\right|_{D\left((-A)^{\beta+\frac{1}{2}}\right)} \leq \rho, \quad d_{H}\left(\hat{y}_{\delta}, \bar{D}\right)>h,
$$

with $\rho, h>0$ to be determined. Once we have fixed $\hat{y}_{\delta}$, for any $x \in B_{\mu, 0}$ we try to construct a continuous mapping $z^{x}:[0, \bar{T}(x)] \rightarrow \mathbb{R}$, with $z^{x}(0)=x$ and $z^{x}(\bar{T}(x))=\hat{y}_{\delta}$, such that (7.4) holds, for a suitable choice of $\bar{T}(x), h$ and $\rho$.

Thanks to Lemma 4.2, for any $\theta>0$ and $\gamma \geq 0$ there exists $T(\gamma, d, \mu, \theta)>0$ such that

$$
u^{x}(t ; 0) \in B_{\theta, \gamma} \cap B_{\theta, \infty}, \quad t>T(\gamma, d, \mu, \theta), \quad x \in B_{\mu, 0} .
$$

Thus, if we set $\gamma=\beta+1 / 2, T_{1}:=T(\beta+1 / 2, d, \mu, \theta)+1$ and

$$
z_{1}^{x}(t):=u^{x}(t ; 0), \quad t \in\left[0, T_{1}\right],
$$

we have that $z_{1}^{x}(0)=x, z_{1}^{x}\left(T_{1}\right) \in B_{\theta, \beta+1 / 2} \cap B_{\theta, \infty}$ and

$$
S_{0, T_{1}}\left(z_{1}^{x}\right)=0 .
$$

Now, for any $T>0$ we define

$$
z_{2}^{x}(t):=\frac{1}{T}(T-t) e^{t A} z_{1}^{x}\left(T_{1}\right), \quad t \in[0, T] .
$$

We have $z_{2}^{x} \in C([0, T] ; H)$ with $z_{2}^{x}(0)=z_{1}^{x}\left(T_{1}\right)$ and $z_{2}^{x}(T)=0$. It is immediate to check that

$$
\mathcal{H}\left(z_{2}^{x}\right)(t)=-G^{-1}\left(z_{2}^{x}(t)\right)\left[\frac{1}{T} e^{t A} z_{1}^{x}\left(T_{1}\right)+B\left(z_{2}^{x}(t)\right)\right], \quad t \in[0, T],
$$

so that

$$
\begin{aligned}
& \left|Q_{\delta}^{-1} \mathcal{H}\left(z_{2}^{x}\right)(t)\right|_{H}^{2} \leq \frac{c_{\delta}}{T^{2}}\left|(-A)^{\beta} G^{-1}\left(z_{2}^{x}(t)\right) e^{t A} z_{1}^{x}\left(T_{1}\right)\right|_{H}^{2} \\
& +c_{\delta}\left|(-A)^{\beta} G^{-1}\left(z_{2}^{x}(t)\right) B\left(z_{2}^{x}(t)\right)\right|_{H}^{2} \\
& =: \frac{c_{\delta}}{T^{2}} I_{1}(t)+c_{\delta} I_{2}(t) .
\end{aligned}
$$

Concerning $I_{1}(t)$, as $z_{2}^{x} \in L^{\infty}\left((0, T) ; D\left((-A)^{\beta+\frac{1}{2}}\right)\right) \subset L^{\infty}\left((0, T) ; L^{\infty}(\mathcal{O})\right)$, by arguing as in the proof of Lemma 5.6. we have

$$
\begin{aligned}
& I_{1}(t) \leq\left|G^{-1}\left(z_{2}^{x}(t)\right) e^{t A} z_{1}^{x}\left(T_{1}\right)\right|_{D(-A)^{\beta}}^{2} \\
& \leq c\left|\frac{1}{g\left(z_{2}^{x}(t)\right)}\right|_{H^{2 \beta+1}(\mathcal{O})}^{2}\left|e^{t A} z_{1}^{x}\left(T_{1}\right)\right|_{H^{2 \beta}(\mathcal{O})}^{2} \leq c\left|e^{t A} z_{1}^{x}\left(T_{1}\right)\right|_{D(-A)^{\beta}}^{2},
\end{aligned}
$$


so that

$$
\begin{aligned}
& \int_{0}^{T} I_{1}(t) d t \leq c \sum_{k=1}^{\infty} \int_{0}^{T} \alpha_{k}^{2 \beta} e^{-2 t \alpha_{k}}\left(z_{1}^{x}\left(T_{1}\right)\right)_{k}^{2} d t \\
& =\frac{c}{2} \sum_{k=1}^{\infty}\left(z_{1}^{x}\left(T_{1}\right)\right)_{k}^{2}\left(1-e^{-2 T \alpha_{k}}\right) \alpha_{k}^{2 \beta-1} \\
& \leq c \sum_{k=1}^{\infty}\left(z_{1}^{x}\left(T_{1}\right)\right)_{k}^{2} \alpha_{k}^{2 \beta-1}=c\left|z_{1}^{x}\left(T_{1}\right)\right|_{D\left((-A)^{\beta-\frac{1}{2}}\right)}^{2} \leq c \theta^{2} .
\end{aligned}
$$

Concerning $I_{2}(t)$, we have

$$
\left|z_{2}^{x}(t)\right|_{D\left((-A)^{\beta}\right)}^{2} \leq \sum_{k=1}^{\infty} \alpha_{k}^{2 \beta} e^{-2 t \alpha_{k}}\left(z_{1}^{x}\left(T_{1}\right)\right)_{k}^{2} .
$$

Moreover, as $z_{1}^{x}\left(T_{1}\right) \in B_{\theta, \infty}$

$$
\left|z_{2}^{x}(t)\right|_{L^{\infty}(\mathcal{O})} \leq\left|z_{1}^{x}\left(T_{1}\right)\right|_{L^{\infty}(\mathcal{O})} \leq \theta, \quad t \geq 0 .
$$

Therefore, by arguing as in the proof of Lemma 4.2, we get that $B\left(z_{2}^{x}(t)\right) \in$ $D\left((-A)^{\beta}\right)$, for any $t>0$, and

$$
\left|B\left(z_{2}^{x}(t)\right)\right|_{D\left((-A)^{\beta}\right)}^{2} \leq c \sum_{k=1}^{\infty} \alpha_{k}^{2 \beta-1}\left(z_{1}^{x}\left(T_{1}\right)\right)_{k}^{2} \alpha_{k} e^{-2 t \alpha_{k}}\left(1+\theta^{2 r}\right),
$$

so that, as for $I_{1}(t)$,

$$
\int_{0}^{T} I_{2}(t) d t \leq c \sum_{k=1}^{\infty} \alpha_{k}^{2 \beta-1}\left(z_{1}^{x}\left(T_{1}\right)\right)_{k}^{2}\left(1+\theta^{2 r}\right)=c\left|z_{1}^{x}\left(T_{1}\right)\right|_{D\left((-A)^{\beta-\frac{1}{2}}\right)}^{2}\left(1+\theta^{2 r}\right) .
$$

In view of (A.3), this yields

$$
\int_{0}^{T}\left|Q_{\delta}^{-1} \mathcal{H}\left(z_{2}^{x}\right)(t)\right|_{H}^{2} d t \leq c_{\delta} \theta^{2}\left(\frac{1}{T^{2}}+\theta^{2 r}+1\right) .
$$

Therefore, if we take $T_{2}=1$, we can find $\bar{\theta}>0$ small enough such that

$$
S_{0, T_{2}}^{\delta}\left(z_{2}^{x}\right) \leq \frac{\eta}{3}
$$

and in correspondence of such $\bar{\theta}$ we can take $T_{1}=T(\beta+1 / 2, d, \mu, \bar{\theta})$.

Next, due to the definition of $S^{\delta}$ and $V_{\delta}$, we can find $z_{3}:\left[0, T_{3}\right] \rightarrow D\left((-A)^{\beta+\frac{1}{2}}\right)$ such that $z_{3}(0)=0$ and $z_{3}\left(T_{3}\right)=y_{\delta}$, with

$$
S_{0, T_{3}}^{\delta}\left(z_{3}\right) \leq V_{\delta}\left(y_{\delta}\right)+\frac{\eta}{3} .
$$

Finally, we want to construct $z_{4}:\left[0, T_{4}\right] \rightarrow H$ with $z_{4}(0)=y_{\delta}$ and $z_{4}\left(T_{4}\right)=\hat{y}_{\delta}$ such that

$$
S_{0, T_{4}}^{\delta}\left(z_{4}\right) \leq \frac{\eta}{3}
$$

We define

$$
z_{4}(t):=e^{t A} y_{\delta}+\frac{t}{T}\left(e^{(T-t) A} \hat{y}_{\delta}-e^{t A} y_{\delta}\right)
$$


Clearly $z_{4}(0)=y_{\delta}$ and $z_{4}(T)=\hat{y}_{\delta}$. Moreover, it is immediate to check that

$$
\mathcal{H}\left(z_{4}\right)(t)=G^{-1}\left(z_{4}(t)\right)\left[\frac{1}{T}\left(e^{(T-t) A} \hat{y}_{\delta}-e^{t A} y_{\delta}\right)-\frac{2 t}{T} A e^{(T-t) A} \hat{y}_{\delta}-B\left(z_{4}(t)\right)\right] .
$$

As $z_{4} \in L^{\infty}\left(0, T ; D\left((-A)^{\beta+\frac{1}{2}}\right)\right)$, by reasoning as above for $z_{1}^{x}$, we have

$$
\begin{aligned}
& \left|Q_{\delta}^{-1} \mathcal{H}\left(z_{4}\right)(t)\right|_{H}^{2} \leq \frac{c_{\delta}}{T^{2}}\left|(-A)^{\beta}\left(e^{(T-t) A} \hat{y}_{\delta}-e^{t A} y_{\delta}\right)\right|_{H}^{2} \\
& +c_{\delta}\left|(-A)^{1+\beta} e^{(T-t) A} \hat{y}_{\delta}\right|_{H}^{2}+c_{\delta}\left|B\left(z_{4}(t)\right)\right|_{D\left((-A)^{\beta}\right)}^{2}=: I_{1}(t)+I_{2}(t)+I_{3}(t) .
\end{aligned}
$$

For the first term $I_{1}(t)$, we have

$$
I_{1}(t) \leq \frac{c}{T^{2}}\left|(-A)^{\beta} e^{(T-t) A}\left(\hat{y}_{\delta}-y_{\delta}\right)\right|_{H}^{2}+\frac{c}{T^{2}}\left|(-A)^{\beta}\left(e^{(T-t) A} y_{\delta}-e^{t A} y_{\delta}\right)\right|_{H}^{2} .
$$

We have

$$
\begin{aligned}
& \int_{0}^{T}\left|(-A)^{\beta} e^{(T-t) A}\left(\hat{y}_{\delta}-y_{\delta}\right)\right|_{H}^{2} d t \\
& \leq c \sum_{k=1}^{\infty} \alpha_{k}^{(2 \beta-1)^{+}}\left(\hat{y}_{\delta}-y_{\delta}\right)_{k}^{2} \alpha_{k} \int_{0}^{T} e^{-2 t \alpha_{k}} d t \leq c\left|\hat{y}_{\delta}-y_{\delta}\right|_{D\left((-A)^{\left(\beta-\frac{1}{2}\right)^{+}}\right)}^{2}
\end{aligned}
$$

Moreover, for $t<T / 2$

$$
\begin{aligned}
& \left|(-A)^{\beta}\left(e^{(T-t) A} y_{\delta}-e^{t A} y_{\delta}\right)\right|_{H}^{2}=\sum_{k=1}^{\infty} \alpha_{k}^{2 \beta}\left(y_{\delta}\right)_{k}^{2} e^{-2 t \alpha_{k}}\left(1-e^{-2(T-2 t) \alpha_{k}}\right)^{2} \\
& \leq c \sum_{k=1}^{\infty} \alpha_{k}^{2 \beta+1}\left(y_{\delta}\right)_{k}^{2} \alpha_{k} e^{-2 t \alpha_{k}}(T-2 t)^{2}
\end{aligned}
$$

and, analogously, for $t>T / 2$

$$
\left|(-A)^{\beta}\left(e^{(T-t) A} y_{\delta}-e^{t A} y_{\delta}\right)\right|_{H}^{2} \leq c \sum_{k=1}^{\infty} \alpha_{k}^{2 \beta+1}\left(y_{\delta}\right)_{k}^{2} \alpha_{k} e^{-2(T-t) \alpha_{k}}(2 t-T)^{2},
$$

so that

$$
\begin{aligned}
& \int_{0}^{T}\left|(-A)^{\beta}\left(e^{(T-t) A} y_{\delta}-e^{t A} y_{\delta}\right)\right|_{H}^{2} d t \\
& \leq c T^{2} \sum_{k=1}^{\infty} \alpha_{k}^{2 \beta+1}\left(y_{\delta}\right)_{k}^{2} \alpha_{k}\left(\int_{0}^{\frac{T}{2}} e^{-2 t \alpha_{k}} d t+\int_{\frac{T}{2}}^{T} e^{-2(T-t) \alpha_{k}} d t\right) \\
& \leq c T^{2}\left|\sqrt{I-e^{2 T A}}(-A)^{\beta+\frac{1}{2}} y_{\delta}\right|_{H}^{2} .
\end{aligned}
$$

Therefore, we can conclude

$$
\int_{0}^{T} I_{1}(t) d t \leq \frac{c}{T^{2}}\left|\hat{y}_{\delta}-y_{\delta}\right|_{D\left((-A)^{\left(\beta-\frac{1}{2}\right)^{+}}\right)}^{2}+c\left|\sqrt{I-e^{2 T A}}(-A)^{\beta+\frac{1}{2}} y_{\delta}\right|_{H}^{2} .
$$


For the second term $I_{2}(t)$, we have

$$
\left|(-A)^{1+\beta} e^{(T-t) A} \hat{y}_{\delta}\right|_{H}^{2}=\sum_{k=1}^{\infty} \alpha_{k}^{2 \beta+1}\left(\hat{y}_{\delta}\right)_{k}^{2} e^{-2(T-t) \alpha_{k}} \alpha_{k},
$$

so that

(A.8)

$$
\begin{aligned}
& \int_{0}^{T} I_{2}(t) d t=\sum_{k=1}^{\infty} \alpha_{k}^{2 \beta+1}\left(\hat{y}_{\delta}\right)_{k}^{2} \alpha_{k} \int_{0}^{T} e^{-2 t \alpha_{k}} d t=\frac{1}{2}\left|\sqrt{I-e^{2 T A}}(-A)^{\beta+\frac{1}{2}} \hat{y}_{\delta}\right|_{H}^{2} \\
& \leq\left|\hat{y}_{\delta}-y_{\delta}\right|_{D\left((-A)^{\beta+\frac{1}{2}}\right)}^{2}+\left|\sqrt{I-e^{2 T A}}(-A)^{\beta+\frac{1}{2}} y_{\delta}\right|_{H}^{2} .
\end{aligned}
$$

Finally, for the third term $I_{3}(t)$, we notice that $z_{4}(t) \in D\left((-A)^{\left.\beta+\frac{1}{2}\right)}\right)$, for any $t \geq 0$ and

$$
\left|z_{4}(t)\right|_{D\left((-A)^{\beta+\frac{1}{2}}\right)} \leq c\left(\left|y_{\delta}\right|_{D\left((-A)^{\beta+\frac{1}{2}}\right)}+\left|\hat{y}_{\delta}\right|_{D\left((-A)^{\beta+\frac{1}{2}}\right)}\right) .
$$

In particular, as $\beta+1 / 2>d / 4$, we have that

$$
\left|z_{4}(t)\right|_{L^{\infty}(\mathcal{O})} \leq c\left|z_{4}(t)\right|_{D\left((-A)^{\beta+\frac{1}{2}}\right)} \leq c\left(\left|y_{\delta}\right|_{D\left((-A)^{\beta+\frac{1}{2}}\right)}+\left|\hat{y}_{\delta}\right|_{D\left((-A)^{\beta+\frac{1}{2}}\right)}\right) .
$$

By arguing as in the proof of Lemma 4.2, due to (2.27) and (2.28) we have that $B\left(z_{4}(t)\right) \in D\left((-A)^{\beta+\frac{1}{2}}\right)$, for any $t \geq 0$ and

$$
\left|B\left(z_{4}(t)\right)\right|_{D\left((-A)^{\beta+\frac{1}{2}}\right)} \leq c\left(1+\left|y_{\delta}\right|_{D\left((-A)^{\beta+\frac{1}{2}}\right)}^{r+1}+\left|\hat{y}_{\delta}\right|_{D\left((-A)^{\beta+\frac{1}{2}}\right)}^{r+1}\right) .
$$

This implies

$$
\begin{aligned}
& \int_{0}^{T} I_{3}(t) d t \leq c\left(1+\left|y_{\delta}\right|_{D\left((-A)^{\beta+\frac{1}{2}}\right)}^{2(r+1)}+\left|\hat{y}_{\delta}\right|_{D\left((-A)^{\beta+\frac{1}{2}}\right)}^{2(r+1)}\right) T \\
& \leq c T\left(1+\left|y_{\delta}\right|_{D\left((-A)^{\beta+\frac{1}{2}}\right)}^{2(r+1)}\right)+c T\left|\hat{y}_{\delta}-y_{\delta}\right|_{D\left((-A)^{\beta+\frac{1}{2}}\right)}^{2(r+1)} .
\end{aligned}
$$

Together with (A.7) and (A.8), this implies

$$
\begin{aligned}
& S_{0, T}^{\delta}\left(z_{4}\right) \leq \frac{c}{T^{2}}\left|\hat{y}_{\delta}-y_{\delta}\right|_{D\left((-A)^{\left(\beta-\frac{1}{2}\right)^{+}}\right)}^{2}+c\left|\sqrt{I-e^{2 T A}}(-A)^{\beta+\frac{1}{2}} y_{\delta}\right|_{H}^{2} \\
& +c\left|\hat{y}_{\delta}-y_{\delta}\right|_{D\left((-A)^{\beta+\frac{1}{2}}\right)}^{2}+c T\left(1+\left|y_{\delta}\right|_{D\left((-A)^{\beta+\frac{1}{2}}\right)}^{2(r+1)}\right)+c T\left|\hat{y}_{\delta}-y_{\delta}\right|_{D\left((-A)^{\beta+\frac{1}{2}}\right)^{2(r+1)}}^{.}
\end{aligned}
$$

Now, if $\left|\hat{y}_{\delta}-y_{\delta}\right|_{D\left((-A)^{\beta+\frac{1}{2}}\right)}<1$, we can fix $T_{4}>0$ such that

$$
\begin{aligned}
& c\left|\sqrt{I-e^{2 T_{4} A}}(-A)^{\beta+\frac{1}{2}} y_{\delta}\right|_{H}^{2}+c T_{4}\left(1+\left|y_{\delta}\right|_{D\left((-A)^{\beta+\frac{1}{2}}\right)}^{2(r+1)}\right) \\
& +c T_{4}\left|\hat{y}_{\delta}-y_{\delta}\right|_{D\left((-A)^{\beta+\frac{1}{2}}\right)}^{2(r+1)}<\frac{\eta}{6},
\end{aligned}
$$


so that

$$
S_{0, T_{4}}^{\delta}\left(z_{4}\right) \leq c\left(\frac{1}{T_{4}^{2}}+1\right)\left|\hat{y}_{\delta}-y_{\delta}\right|_{D\left((-A)^{\beta+\frac{1}{2}}\right)}^{2}+\frac{\eta}{6} .
$$

Therefore, if we take $\rho_{\eta}<1$ such that

$$
\begin{aligned}
& \left|\hat{y}_{\delta}-y_{\delta}\right|_{D\left((-A)^{\beta+\frac{1}{2}}\right)}^{2(r+1)}<\rho_{\eta} \\
& \Longrightarrow c\left(\frac{1}{T_{4}^{2}}+1\right)\left|\hat{y}_{\delta}-y_{\delta}\right|_{D\left((-A)^{\beta+\frac{1}{2}}\right)}^{2}<\frac{\eta}{6},
\end{aligned}
$$

we obtain (A.6).

Hence, collecting all terms, if we fix $\gamma=\beta+1 / 2, \bar{\theta}>0$ such that A.4 holds and $h=\rho_{\eta}$ in (A.1) and if we define

$$
z^{x}(t):= \begin{cases}z_{1}^{x}(t), & t \in\left[0, \hat{T}_{1}\right], \\ z_{2}^{y}\left(t-\hat{T}_{1}\right), & t \in\left[\hat{T}_{1}, \hat{T}_{2}\right], \\ z_{3}\left(t-\hat{T}_{2}\right), & t \in\left[\hat{T}_{2}, \hat{T}_{3}\right], \\ z_{4}\left(t-\hat{T}_{3}\right), & t \in\left[\hat{T}_{3}, \hat{T}_{4}\right],\end{cases}
$$

where

$$
\hat{T}_{i}=\sum_{k=1}^{i} T_{k}
$$

we have that $z^{x} \in C([0, \bar{T}(x)] \rightarrow H$, with

$$
\bar{T}(x):=\hat{T}_{4},
$$

which is independent of $x \in B_{\mu, 0}$, and $z^{x}(0)=x$ and $z^{x}(\bar{T}(x))=\hat{y}_{\delta}$. In particular, in view of (A.1) we obtain (7.3). Moreover, thanks to (A.2), (A.4), (A.5) and (A.6), we have

$$
S_{0, \bar{T}(x)}^{\delta}\left(z^{x}\right)=S_{0, T_{1}}^{\delta}\left(z_{1}^{x}\right)+S_{0, T_{2}}^{\delta}\left(z_{2}^{y}\right)+S_{0, T_{3}}^{\delta}\left(z_{3}\right)+S_{0, T_{4}}^{\delta}\left(z_{4}\right) \leq V_{\delta}\left(y_{\delta}\right)+\eta .
$$

A.2. Proof of Lemma 7.3. Let $\bar{\mu}>0$ such that $B_{\bar{\mu}, 0} \subset D$. As proved in [4, the family $\left\{\mathcal{L}\left(u_{\epsilon}^{x}\right)\right\}_{\epsilon>0}$ satisfies a large deviation principle in $C([0, T] ; H)$, for any $T>0$, with action functional $S_{0, T}^{\delta}$. Thus, in view of Lemma 7.2, for any $\eta>0$ and $\mu \leq \bar{\mu}$, there exists $\epsilon_{0}>0$ such that

$$
\begin{aligned}
& \mathbb{P}\left(\tau_{\epsilon, x}<\bar{T}\right) \geq \mathbb{P}\left(\tau_{\epsilon, x}<\bar{T}(x)\right) \geq \mathbb{P}\left(\sup _{t \in[0, \bar{T}(x)]}\left|u_{\epsilon}^{x}(t)-z^{x}(t)\right|_{H}<h\right) \\
& \geq \exp \left(-\frac{1}{\epsilon}\left(S_{0, \bar{T}(x)}^{\delta}\left(z^{x}\right)+\frac{\eta}{2}\right)\right) \geq \exp \left(-\frac{1}{\epsilon}\left(\inf _{y \in \partial D} V_{\delta}(y)+\eta\right)\right),
\end{aligned}
$$

for any $\epsilon<\epsilon_{0}$ and $x \in B_{\mu, \infty}$. This immediately implies (7.5).

A.3. Proof of Lemma 7.4, We proceed as in [9, proof of Lemma 5.7.19], by using Lemma 4.2 and the compactness of the level sets of $S_{0, T}^{\delta}$, for any $T>0$, proved in [4, Theorem 5.1]. Notice that here the situation is simpler than in [9, Lemma 5.7.19], because we are not proving an estimate uniform with respect to $x \in \bar{G}$, but only one for any fixed $x \in G$. 
A.4. Proof of Lemma 7.5. As for any $x \in D$ and $T>0$ the set $\left\{u^{x}(t) ; t \in\right.$ $[0, T]\} \subset D$ is compact, so that its distance from $\partial D$ is strictly positive. Thus, in order to prove (7.7) we can proceed as in [9, proof of Lemma 5.7.22].

A.5. Proof of Lemma 7.6. For any $t \geq 0$ and $x \in G$ we have

$$
\left|u_{\epsilon, \delta}^{x}(t)-x\right|_{H} \leq\left|u_{\epsilon, \delta}^{x}(t)-e^{t A} x\right|_{H}+\left|e^{t A} x-x\right|_{H} .
$$

Thus, for any $\rho>0$ fixed, we can find $T_{1}(\rho, x)$ such that

$$
\mathbb{P}\left(\sup _{t \in\left[0, T_{2}(\rho, x)\right]}\left|u_{\epsilon, \delta}^{x}(t)-x\right|_{H} \geq \rho\right) \leq \mathbb{P}\left(\sup _{t \in\left[0, T_{1}(\rho, x)\right]}\left|u_{\epsilon, \delta}^{x}(t)-e^{t A} x\right|_{H} \geq \frac{\rho}{2}\right) .
$$

Now, according to (2.31), we can fix $T_{2}(\rho, x) \leq T_{1}(\rho, x)$ such that

$$
\begin{aligned}
& \mathbb{P}\left(\sup _{t \in\left[0, T_{2}(\rho, x)\right]}\left|u_{\epsilon, \delta}^{x}(t)-e^{t A} x\right|_{H} \geq \frac{\rho}{2}\right) \\
& \leq \mathbb{P}\left(\sup _{t \in\left[0, T_{2}(\rho, x)\right]}\left|\int_{0}^{t} e^{(t-s) A} Q_{\delta} G\left(u_{\epsilon, \delta}^{x}(s)\right) d w(s)\right|_{H} \geq \frac{\rho}{4 \sqrt{\epsilon}}\right),
\end{aligned}
$$

and then the conclusion of the lemma follows from the exponential estimates for the stochastic convolution (for a proof see [5, Theorem 3.2]).

\section{REFERENCES}

[1] S. Cerrai, Second order PDE's in finite and infinite dimension. A probabilistic aPPROACH, Lecture Notes in Mathematics Series 1762, Springer-Verlag (2001). MR.1840644 (2002j:35327)

[2] S. Cerrai, Stochastic reaction-diffusion systems with multiplicative noise and non-Lipschitz reaction term, Probability Theory and Related Fields 125 (2003), pp. 271-304. MR.1961346 (2004a:60117)

[3] S. Cerrai, Asymptotic behavior of systems of SPDE's with multiplicative noise, Stochastic Partial Differential Equations and Applications VII, Lecture Notes in Pure and Applied Mathematics 245 (2005), Chapman and Hall/CRC Press, pp. 61-75. MR2227220 (2008e:60187)

[4] S. Cerrai, M. Röckner, Large deviations for stochastic reaction-diffusion systems with multiplicative noise and non-Lipschitz reaction term, Annals of Probability 32 (2004), pp. 11001139. MR2044675 (2005b:60063)

[5] S. Cerrai, M. Röckner, Large deviations for invariant measures of stochastic reactiondiffusion systems with multiplicative noise and non-Lipschitz reaction term, Annales de l'Institut Henri Poincaré (Probabilités et Statistiques) 41 (2005), pp. 69-105. MR2110447 (2005k:60084)

[6] G. Da Prato, J. Zabczyk, Stochastic Equations in Infinite Dimensions, Cambridge University Press, Cambridge (1992). MR1207136 (95g:60073)

[7] G. Da Prato, A. J. Pritchard, J. Zabczyk, On minimum energy problems, SIAM Journal on Control and Optimization 29 (1991), pp. 209-221. MR1088228 (92b:49049)

[8] G. Del Maso, An introduction to Г-convergence, Birkäuser Boston (1993). MR1201152 (94a:49001)

[9] A. Dembo, O. Zeitouni, Large Deviations Techniques and Applications, Second Edition, Springer-Verlag (1998). MR.1619036 (99d:60030)

[10] M.I. Freidlin, A.D. Wentzell, Random Perturbations of dynamical systems, Second edition, Springer-Verlag, New York (1998). MR1652127 (99h:60128)

[11] E. Gautier, Exit from a basin of attraction for stochastic weakly damped nonlinear Schrödinger equations, Annals of Probability 36 (2008), pp. 896-930. MR 2408578 (2009d:60206) 
[12] T. Runst, W. Sickel, Sobolev Spaces of Fractional Order, Nemytskij Operators and Nonlinear Partial Differential Equations, Walter de Gruyter, Berlin, New York (1996). MR.1419319 (98a:47071)

[13] R. Sowers, Large deviations for the invariant measure of a reaction-diffusion equation with non-Gaussian perturbations, Probability Theory and Related Fields 92 (1992), pp. 393-421. MR:1165518 (93h:60043)

[14] H. Triebel, Interpolation Theory, Function Spaces, Differential Operators, NorthHolland, Amsterdam (1978). MR.503903 (80i:46032b)

Department of Mathematics, University of Maryland, College Park, Maryland 20742

Department of Mathematics, University of Maryland, College Park, Maryland 20742 